\title{
Automated Detection and Classification of Cervical Cancer Using Pap Smear Microscopic Images: A Comprehensive Review and Future Perspectives
}

\author{
Shanthi P B, ${ }^{1, *}$ Hareesha $\mathrm{K} \mathrm{S}^{2, *}$ and Ranjini Kudva ${ }^{3}$
}

\begin{abstract}
Computer-aided categorization of smear images has been considered challenging in the past few decades. Cervical cancer is the main cause of mortality among women worldwide and is more prevalent in underdeveloped countries. This disease can be successfully treated, even fully cured, if detected in its early phase. Computerized image analysis methods are primarily of great interest as they provide significant benefits for clinicians with reliable and timely diagnosis of the samples. Dedicated image analysis algorithms provide a mathematical description of the region of interest which provide great support to pathologists for decision-making. In this review, we have outlined state-of-the-art techniques expressed in prominent publications on the computer-assisted diagnostic system for cancer detection. By utilizing the domain aspects of cervical cancer, suitable methods and techniques are explored and presented. This review also presents knowledge to assess the methodology used in the literature and emphasized some of the inadequacies and weaknesses in the reviewed methods. The study accentuates the future directions pertinent to the development of a cost-effective, automated disease classification system that should be a significant advantage for countries with limited resources and treatment services.
\end{abstract}

Keywords: Enhancement; Classification; Cellular Feature Extraction; Segmentation; Uterine Cancer.

Received: 29 October 2021; Revised: 08 January 2022; Accepted: 16 January 2022.

Article type: Review article.

\section{Introduction}

Uterine cancer is an abnormal, proliferating growth of cells. Malignant cells can spread to neighboring tissues and other organs of the body. Cervical cancer originates in the tissue of the cervical region, which is the bottommost part of the uterus. The infected uterine cells grow and multiply with abnormal cell cycles. ${ }^{[1]}$ Since it is slow-growing cancer, women with early-stage of cervical cancer do not have any symptoms until it becomes invasive cancer and spreads to other organs of the body. As the disease has a long pre-malignant phase, the diagnosis of the disease at its early stage is completely curable

\footnotetext{
${ }^{I}$ Department of Computer Science and Engineering, Manipal Institute of Technology, Manipal Academy of Higher Education, Manipal, Karnataka, India,576104.

2 Department of Computer Application, Manipal Institute of Technology, Manipal Academy of Higher Education, Manipal, Karnataka, India,576104.

${ }^{3}$ Department of Pathology, Kasturba Medical College, Manipal Academy of Higher Education, Manipal, Karnataka, India, 576104.

*E-mail: shanthi.moorkoth@manipal.edu (Shanthi P B);

hareesh.ks@manipal.edu (Hareesha K S)
}

and preventable. ${ }^{[1]}$

In India, this disease is the most common cancer among women between the age of 15 and $44 .^{[1]}$ The recent results from GLOBOCAN 2018 produced by "International Agency for Research on Cancer" assessed breast, cervix, ovary, oral cavity, and lung cancers as the top five types found in females prevalent in India. ${ }^{[2]}$ Fig. 1 shows the projected incidence and mortality rate of these top five cancers among females of all ages in India. ${ }^{[2]}$ At present, breast cancer is the leading type among the top five, and cervix uterine cancer in the second position, which is shown in Fig. 2. According to the GLOBOCAN 2018 data, new cervical cancer cases registered in India about 96,000, which were 123,000 in 2012 and 134,000 in $2008 .^{[2]}$ The mortality rate of cancer was 73,000 in 2008 , reduced to around 60,000 in 2018 . The drastic reduction in incidence and mortality rates is due to the awareness created by the effective screening programs, and the importance of primary diagnosis and treatment programs used for abnormality detection.

According to the current statistics of GLOBOCAN 2020, ${ }^{[3]}$ breast cancer stands first among women followed by cervix cancer. The percentage of incidence of breast cancer has come 


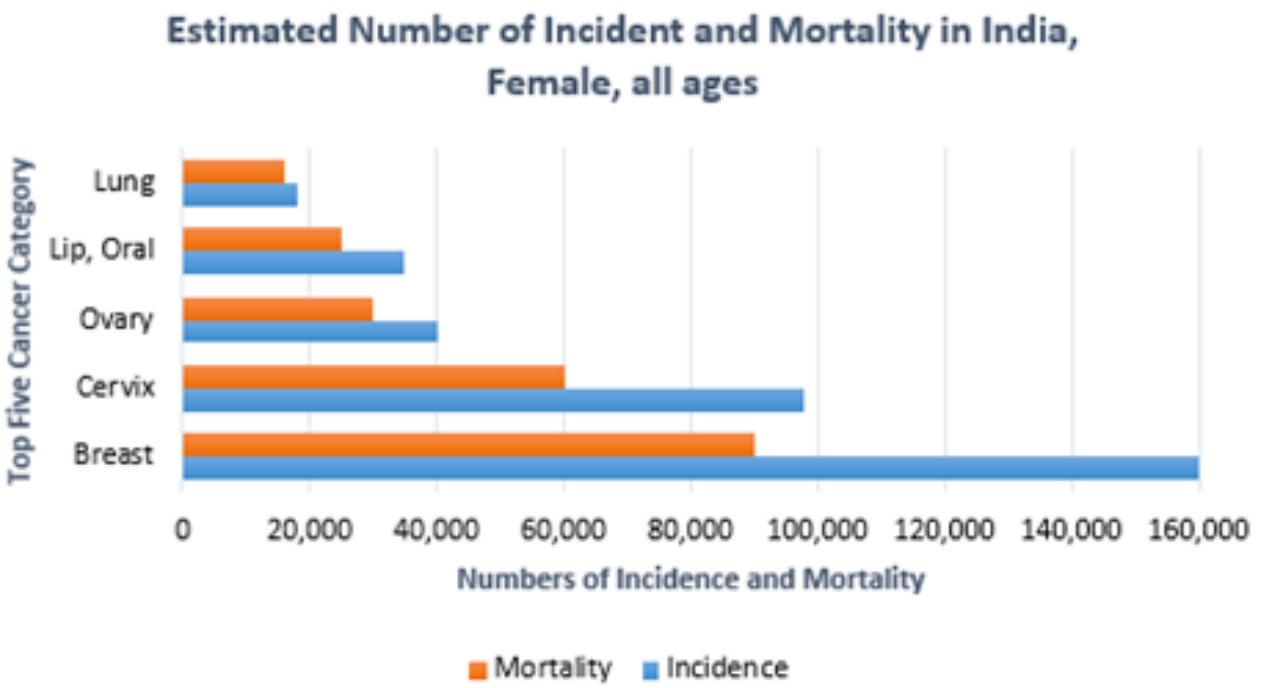

Fig. 1 Incidence and Mortality Rates of Top 5 Cancers in India in Female Category of all Ages. Reproduced with permission from [2], Copyright 2018 American Cancer Society.

down from $28 \%$ to $23 \%$ and cervical cancer incidence to $9 \%$ from $17 \%$, as shown in Fig. 3 . This is a great reduction in cervical cancer incidences among the female population in India.

Papanicolau Smear test is a pap smear test named after Dr. Papanicolau. It is one of the widely used conventional screening tests which helps to detect changes in the cells of the cervix. The screening method helps to identify abnormality at its early pre-cancerous stage. In developed countries, routine and compulsory screening for cervical cancer helped in reducing the incidence and mortality rates caused by this disease. But in developing countries, lack of resources, insufficient knowledge, and consequences of the disease increase the incidence and mortality rates of cancer and still remains a serious threat among women. Automated devices that are designed to screen Pap Smears had some limitations, which include reduced sensitivity (ability to correctly identify the sample with the disease), uncertainty over costeffectiveness, and failure to detect cases of initial-stage abnormalities. In 2003, the "U.S. Preventive Services Task Force (USPSTF)" expressed inadequate evidence for the routine usage of automated devices. ${ }^{[4]}$ In 2005, the "National Coordinating Centre for Health Technology Assessment" concluded that the performance, impact, and cost were still insufficient for the recommendation of automated systems. ${ }^{[4]}$ In 2006, "Screening for Cervical Cancer: an overview" from "Obstetrics and Gynecology of India" stated that a lot of studies were required to evaluate this technology to incorporate into a screening program. ${ }^{[5]}$ In 2011, "Manual Assessment versus

\section{Number of New Cases in 2018 , Females, All Ages}

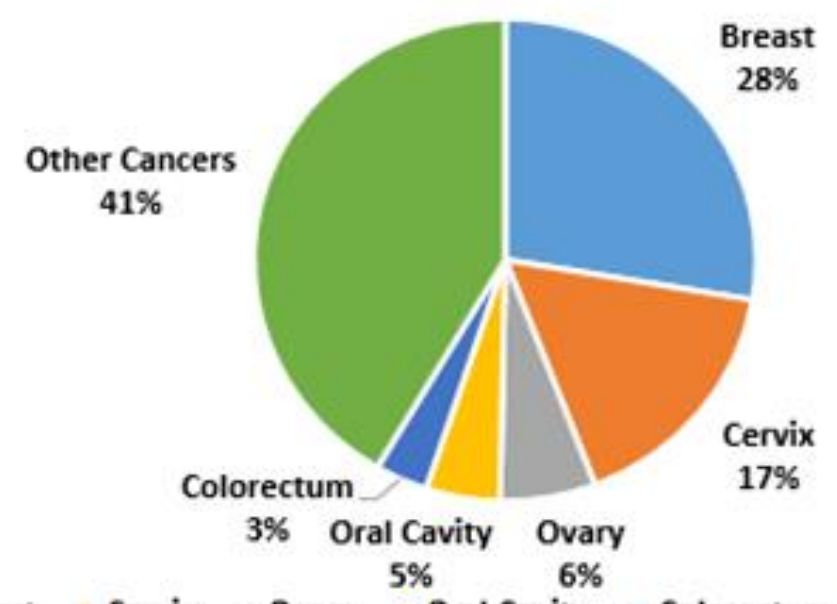

= Breast $\equiv$ Cervix $=$ Ovary $=$ Oral Cavity $\equiv$ Colorectum "Other Cancers

Fig. 2 New Cancer Cases (Incidence) in 2018 among Females Category in India of all ages. Reproduced with permission from [2], Copyright 2018 American Cancer Society. 


\section{NUMBER OF NEW CASES IN 2020, FEMALES, ALL AGES}

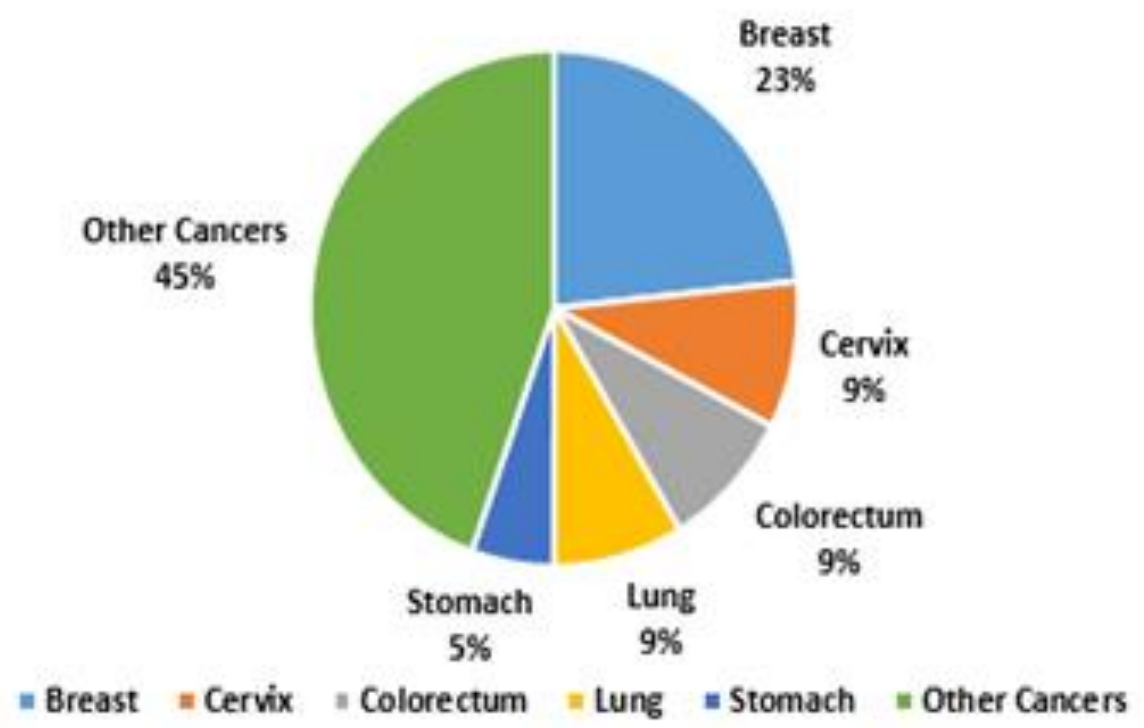

Fig. 3 New Cancer Cases (Incidence) in 2020 among Female Category in India of all Ages. Reproduced with permission from [3], Copyright 2018 American Cancer Society.

Automated Reading in Cytology (MAVARIC)" - a Journal from Health Technology Assessment concluded that low sensitivity and high device cost suggested no justification for the use of screening purposes. ${ }^{[6]}$

Manual screening vs. automated systems has been an open problem and remains unsolved. Manual screening of cell images is laborious and time-consuming as each image within a smeared slide has to be analyzed by seeing through the microscope by the pathologists for disease diagnosis. Large numbers of samples have to be examined during the screening of the mass population, which is time-consuming and requires skilled technicians. Technology advancement in medical imaging has enhanced the quality of medical images leading to better contributions to the early diagnosis of diseases. Computer-assisted commercial products such as AutpPap300, PapNet, AutoCyte, Cytoanalyzer, CYBEST, etc., were designed to automate the screening process, but most of the devices showed limitations by producing too many falsepositive rates, uncertainty over cost-effectiveness, and also a failure in detecting low-grade abnormalities. ${ }^{[6,7]}$ In spite of the availability of advanced automated systems for smear analysis, underdeveloped and middle-income countries with high occurrence and death rates of cervical cancer are inadequate in the use of automated systems because of the high cost and maintenance. ${ }^{[7]}$ So the development of a low-cost and effective automated screening tool has become a major research area that helps Pathologists for faster analysis and reliable diagnosis of samples.

In this review, an efficient classification algorithm is proposed for a computer-assisted disease diagnosis tool. The success of the computational approach depends on three levels of processing. In the first level which is the initial level of processing, noise removal, and region of interest enhancement are performed on the images. In the middle level of processing, accurate localization and segmentation of cell nuclei and textural feature extraction are achieved for quantitative analysis of cell images. As a part of high-level processing, classification and decision making is performed to identify the different phases of the disease as five classes ( Normal, Mild, Moderate, Severe, and Carcinoma) ${ }^{[5,6]}$ based on World Health Organization (WHO) terminology, four class (Normal, CIN1, CIN2, and CIN3) $)^{[5,6]}$ based on Cervical Intraepithelial Neoplasia (CIN) terminology, three class (Normal, Low Grade and High Grade $)^{[5,6]}$ based on Bethesda system (TBS) terminology and two class (Normal and Abnormal) and shown in Table 1.

Table 1. WHO, CIN, and Bethesda System Terminologies for Cancer Grading.

\begin{tabular}{|c|c|c|}
\hline $\begin{array}{c}\text { WHO } \\
\text { Terminology }\end{array}$ & $\begin{array}{c}\text { CIN } \\
\text { Terminology }\end{array}$ & Bethesda Terminology (TBS) \\
\hline $\operatorname{Mild}^{[5,6]}$ & CIN 1 & $\begin{array}{c}\text { Low-Grade Squamous } \\
\text { Intraepithelial Lesion (LSIL) }^{[5]}\end{array}$ \\
\hline Moderate ${ }^{[5,6]}$ & CIN 2 & \multirow{3}{*}{$\begin{array}{c}\text { High-Grade Squamous } \\
\text { Intraepithelial Lesion (HSIL) }\end{array}$} \\
\hline Severe $^{[5,6]}$ & CIN 3 & \\
\hline Carcinoma $^{[5,6]}$ & CIN 3 & \\
\hline $\begin{array}{c}\text { Invasive } \\
\text { Cancer }^{[5,6]}\end{array}$ & $\begin{array}{l}\text { Invasive } \\
\text { Cancer }\end{array}$ & Invasive Cancer \\
\hline
\end{tabular}

\section{Cell biology and cervical cytology}

The cell is the smallest structure of the body that performs all the processes that define life. Cytology or cellular biology deals with the study of cells, their physiological properties, structure, and the organelles inside the cell, their interaction 

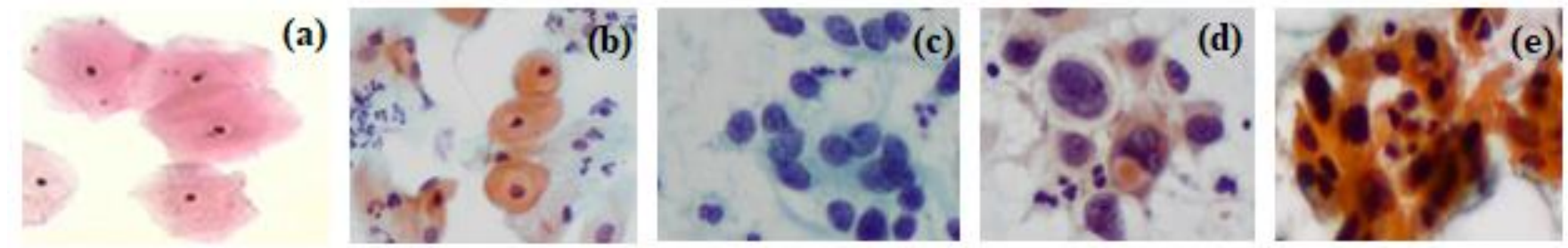

Fig. 4 Pap Smear Cervical Intraepithelial Neoplasia (CIN) Slide Images (a) Normal, (b) CIN1, (c) CIN2, (d) CIN3, and (e) Invasive Cancer. Reproduced with permission from Kasturba Medical College (KMC), Department of Pathology, Manipal, India. (IEC 472/2013).

with the environment, cell cycle, and cell death. The branch of study about cellular diseases by observing the cellular variations in the form of size, shape, color, and texture for the diagnosis of the disease is called cytopathology. The three main parts of the cell are the nucleus, the cytoplasm, and the membrane. The control part of the cell is the nucleus, and the chromatins, which are a thread-like structure inside the nucleus contain deoxyribonucleic acid (DNA) and genetic materials. There is one nucleolus, which is the dense region of ribonucleic acid (RNA) in the nucleus. ${ }^{[8]}$ Normal cells grow, divide to make new cells, and die when they complete their task. Abnormality causes when the cells start to grow and form new abnormal cells without dying. This abnormality causes changes in the appearance of the cell in the form of size, shape, texture, alignment, and color. ${ }^{[9]}$ In the CIN1 or Mild category, deformity starts with little nuclear amplification and ample cytoplasmic region. It has a highly "keratinized cytoplasm" with distinct cell boundaries.

Cells with distinct and dense cytoplasm also define the abnormality of CIN1. In the CIN2 or moderate category, cells occur individually or cluttered and have large cytoplasm areas and occasionally the cytoplasm is heavily "keratinized". More than one-half of the area in the cell is occupied by the nucleus. Nuclear amplification is greater than normal cell nuclei, and nuclear edges are distorted with distinct Hyperchromatism. In CIN3 (HSIL or Severe and Carcinoma cells) most of the abnormalities are the same as in CIN2, but two-thirds of the area of the cell is occupied by the nucleus. The dissimilarity in size and shape of the nucleus with asymmetrical chromatin content and overlapped cluttered clusters of cells is highly noticeable. Invasive cancer cells have enlarged irregular nuclei, distinct multiple nucleoli, and hyperchromatic nuclei that overlap and mold with each other. ${ }^{[10,11]}$ The appearance of the cell images of different grades is shown in Fig. 4. These images were collected from Kasturba Medical College (KMC), Department of Pathology, Manipal, India, and ethical committee approval was obtained from KMC experts for use this data.

The morphological changes that the nucleus and cytoplasm undergo during the disease progression phase from benign to malignancy are very distinct for diagnosis. The nucleus deformations of the cell are more prominent than the cytoplasm for disease classification and diagnosis because of the darker intensity, larger nucleus-to-cytoplasmic ratio, and coarse texture due to the irregular chromatin distribution ${ }^{[1,12]}$ as shown in Fig. 5.
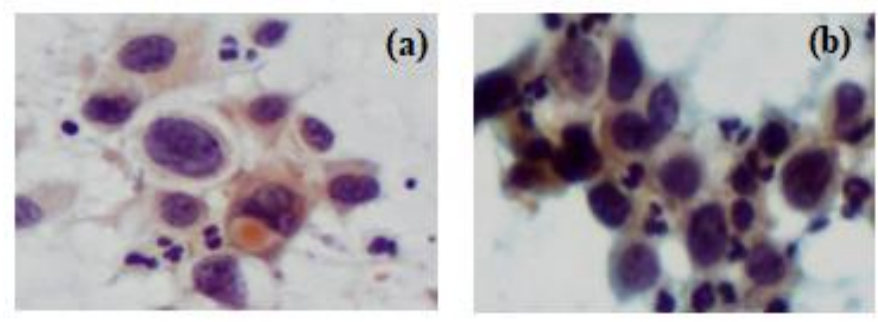

Fig. 5 Hyperchromatic Nucleus and Fused Cytoplasmic Feature of Uterine Cervix Cancer Slide Images (a) High-Grade Cancer Cell Image and (b) Invasive Cancer Cell Image. Reproduced with permission from Kasturba Medical College (KMC), Department of Pathology, Manipal, India. (IEC 472/2013).

\section{Image dataset}

Computer-aided diagnosis requires a considerable amount of labeled data for precise and accurate validation that results in efficient classification accuracy. Various studies on cancer screening and diagnosis are based on the publicly available dataset as well as individually collected data. A proper baseline benchmark dataset is very essential as a major part of the research work lies in comparison and validation, which can be executed only with the authenticated dataset. Herlev Dataset ${ }^{[13]}$ is a publicly available dataset that was developed by the Pathology Department at Herlev University ${ }^{[13]}$ combined with the Department of Automation at the Technical University of Denmark (DTU) ${ }^{[13]}$ and is used by many students and researchers to test and compare their own classification methods. This dataset consists of 917 images of pap smear cervical cells, ${ }^{[13]}$ collected meticulously by the cytotechnicians using a microscope with a magnification of 0.201 $\mu \mathrm{m} /$ pixel. $^{\left[{ }^{[13]}\right.}$ Individually collected dataset from KMC, Department of Pathology, Manipal, India, which are based on single-cell images of pap smear cells of $20 \mathrm{X}$ magnification, is also highlighted and used in the review study and shown in Figs. 4, 5, 8, and 10. An overview of the publicly available pap smear datasets such as ISBI2014 and ISBI2015 datasets (International Biomedical Imaging Symposium), CERVIX, CRIC Cervix, SIPakMed, and DHB (Digital Human Body) ${ }^{[14,15]}$ are given in Table 2 and Figs. 6, 7, and 8 display the sample images of some of the publicly available and individually collected KMC datasets. ${ }^{[16,17]}$ 

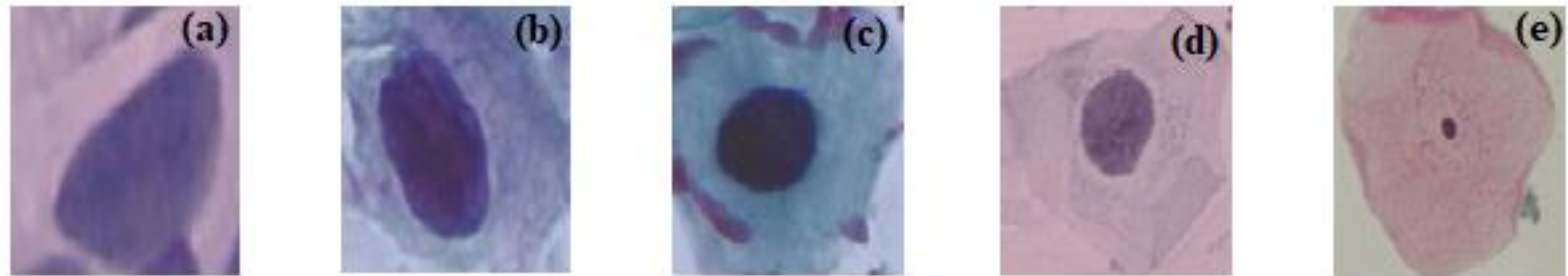

Fig. 6 Pap-Smear Benchmark Herlev Dataset Cervix Cell Images (a) Carcinoma Cell, (b) Severe Cell, (c) Moderate Cell, (d) Mild Cell, and (e) Normal Cell. Reproduced with permission from ${ }^{[13]}$, Copyright (C) 2011 MDE-Lab.
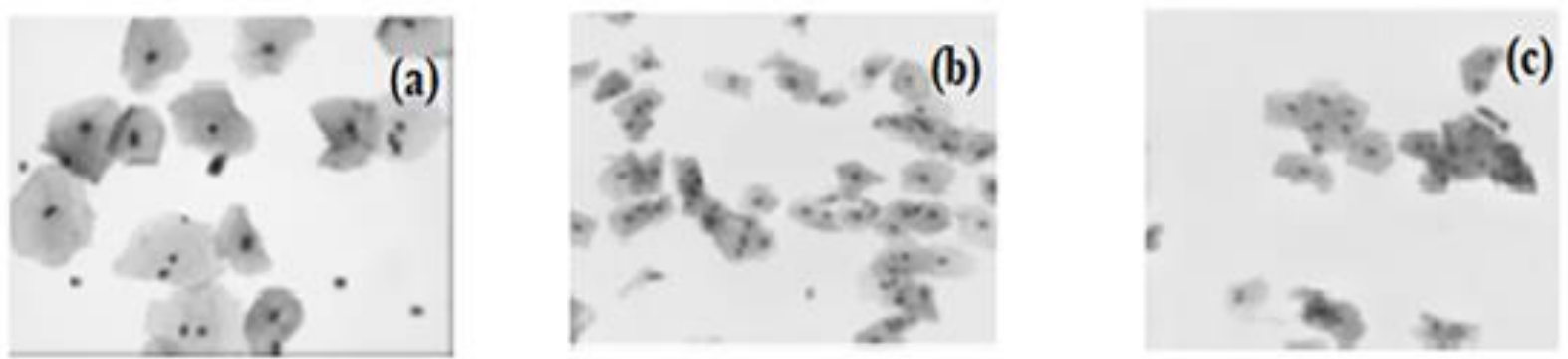

Fig. 7 Pap Smear Cervix Cell Slide Images (a) CERVIX93 Dataset Image, (b) ISBI14 Dataset Image, and (c) ISBI15 Dataset Image. Reproduced with permission from ${ }^{[14]}$.
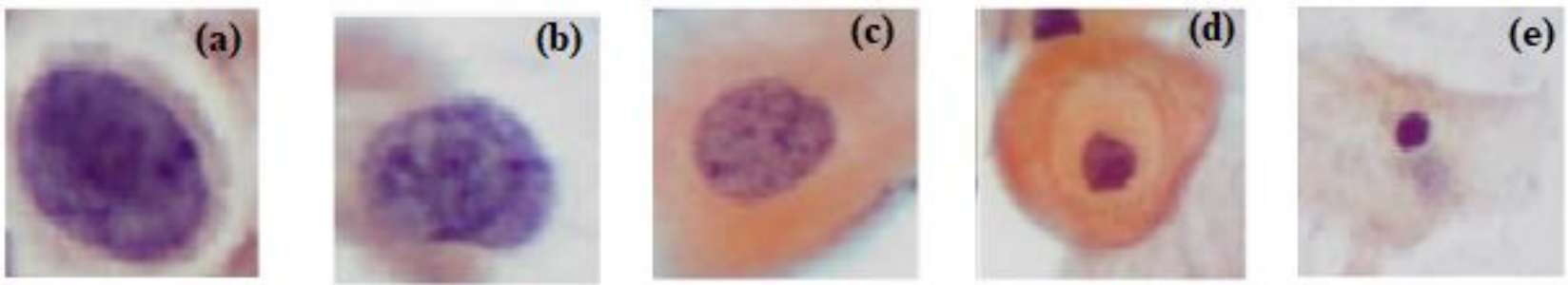

Fig. 8 Pap Smear Cervix Cell Images collected from the Department of Pathology, Kasturba Medical College, Manipal, Karnataka (a) Carcinoma Cell, (b) Severe Cell, (c) Moderate Cell, (d) Mild Cell and (e) Normal Cell. Reproduced with permission from Kasturba Medical College (KMC), Department of Pathology, Manipal, India. (IEC 472/2013).

Table 2. Publicly available Pap smear dataset.

\begin{tabular}{cccc}
\hline Data Used & Year & No. of Images & Description \\
\hline DTU/Herlev Dataset $^{[13]}$ & 2003 & 500 & Single Cell Images \\
DTU/Herlev Dataset $^{[13]}$ & 2005 & 917 & Single Cell Images \\
ISBI14 $^{[14,15]}$ & 2014 & $961(16 \mathrm{EDF}+945$ Synthetic $)$ & Slide Images \\
ISBI15 $^{[14,15]}$ & 2015 & $37(17 \mathrm{EDF}($ each with 20 FOVs $)$ & Slide Images \\
CERVIX $^{[14,15]}$ & 2018 & $113(93 \mathrm{EDF}($ each with 20 FOVs $)$ & Slide Images \\
CRIC Cervix $^{[16]}$ & 2021 & 400 with 11,534 classified cells, 0.228 $\mu \mathrm{m} /$ pixel & Slide Images \\
SIPakMed $^{[16]}$ & 2018 & 966 with 4049 classified cells & Slide Images \\
DHB $^{[16]}$ & 2021 & 500 with 5414 classified cells & Whole Slide Image
\end{tabular}

\section{Computational approaches for smear cytology}

The aim of computer-assisted pap smear image analysis is to efficiently support the detection of possible suspicious images. Medical Image analysis includes different techniques and processing methods that aid abundantly in clinical analysis and interventions of medical images. Recently, in medical domains, large volumes of data and images can be analyzed and interpreted by using different machine learning algorithms These algorithms include statistical, probabilistic, and different optimization techniques and allow the computer to learn and analyze samples in a better way. Pap smear analysis system consists of stages such as image enhancement which aids in the improvement of the region of interest, segmentation helps in the extraction of the region of interest, feature extraction which describes the feature of the extracted region of interest and finally the classification, which is the most important phase that determines whether a sample is malignant or normal. This review includes literature related to the application of image investigation, computerized diagnosis, and classification of malignant cells from cellular images. 


\subsection{Image enhancement}

The goal of image preprocessing is to emphasize certain structures for subsequent analysis of samples. Detection of the lesion in microscopic cervical cell images is a crucial task due to several facts. Pre-processing is considered an essential step before any image analysis process, which makes the image more relevant by removing irrelevant information. ${ }^{[18,19]}$ The main goal is to suppress the noise and preserve the integrity of edges and the other detailed information in the image for effective classification. Image enhancement deals with image quality and the factors that influence the quality of an image include contrast, brightness, spatial resolution, and noise. ${ }^{[19]}$ Artifacts included in the samples such as blood-smeared cells, overlapped cells, crumpled cells, and the presence of bacteria obstruct the cell segmentation, feature extraction, and classification process. Image quality and enhancement play a vital role in efficient image segmentation, feature extraction, and classification phase. Filtering one of the portions of image enhancement removes unwanted noise and also enhances certain information in the image. ${ }^{[18,19]}$ Microscopic images are degraded mostly with Gaussian and Poisson noise. Wiener filter, average filter, min-max filter, and median filter are some of the filters used for enhancing a region that has to be extracted in the pap smear cell images..$^{[19,20]}$ Different enhancement algorithms are reviewed and identified as the most suitable enhancement method for cervical cell images.

A comparative analysis of various image enhancement techniques has been conducted on spatial, frequency, and Fuzzy domain methods ${ }^{[21-23]}$ and identified that Fuzzy logic and histogram-based methods are showing effective results. As a future enhancement in the proposed method, the author suggests automating the evaluation of the K factor of Fuzzybased enhancement using the ant colony optimization to represent the image in a more efficient manner. ${ }^{[22-24]} \mathrm{A}$ study on $\mathrm{YCbCr}$ ( luminance $(\mathrm{Y})$, chroma blue $(\mathrm{Cb})$, and chroma red $(\mathrm{Cr})$ ) color space methods is presented, and proposed the HSI reference model for enhancing $\mathrm{Y}, \mathrm{Cb}$, and $\mathrm{Cr}$ components. ${ }^{[25-27]}$ Gaussian noise has been added with various standard deviations and achieved satisfactory results. Magudeeswaran et al. propose a method of fuzzy logic-based contrast enhancement. In the proposed method, the first stage fuzzy histogram is computed to handle the vagueness of the gray level value. Based on the median value, in the next stage, the fuzzy histogram is distributed into two sub histograms and independently equalized to preserve the brightness of the image. ${ }^{[28]}$

Deepa et al. study Poisson noise denoising on pap smear images. The study reveals that the Poisson noise could be effectively removed using an adaptive Wiener filter. Adaptive Wiener filter can denoise images effectively even in the variation of image characteristics at different locations and produce an effective result on image enhancement. ${ }^{[29]}$ Vendhan et al. propose a two-stage technique. In that, the membership value for a pixel is identified prior to enhancement using the Fuzzy trapezoidal membership function, and the highest membership function value for a pixel is enhanced. Non-linear contrast algorithms are used to spot the structural variations in the cell cytoplasm and nucleus portions. The proposed method successfully enhances the region of interest of cervical cells, along with the overall enhancement of the image. ${ }^{[20,23]}$

Tang et al. suggest a new approach to bi-histogram equalization ${ }^{[18,30]}$ to enhance grayscale images. This method splits the input histogram into a pair of small histograms. Over-enhancement of the image is controlled by clipping the histogram, and the output has been equalized and combined to obtain the resultant image. The proposed method by the authors outperforms other histogram-based enhancement methods. ${ }^{[30]}$ Guan et al. combine intensity and color-based information to enhance the cell nuclei. Morphological reconstruction is used to extract the regional maxima of a cell sample and extracted the true nuclei using geometrical features. The nuclei portion is made more obvious through color intensity information. The intensity-based color distribution of the nuclei enhancement model performs well and successfully enhanced the region of interest. ${ }^{[22,24]}$

Vidyasaraswathi et al. address the issues of various image enhancement methods and analyze several algorithms on histogram equalization suitable for medical images. ${ }^{[31]}$ Authors adopt histogram equalization algorithms to improve the image contrast and resolution to preserve textural information and reduce image blurring. They also address "Iterative Dynamic Histogram Equalization, Dualistic Sub Image Histogram Equalization and Brightness based Bi-Histogram Equalization" for effective image preprocessing, especially in medical images..$^{[18,19,31]}$ A new approach is presented using the algorithm "Brightness Preserved Fuzzy Histogram Equalization combined with Homomorphic Filtering". This avoids false contouring in the region with high similarities in intensities or regions with a rapid change of intensities and maintained mean brightness and preserved the image's appearance. The fuzzification used in the algorithm improves the crisp definition to alleviate contouring in highly correlated areas. $^{[23,31]}$

Kumbhar et al. work on image enhancement problems, especially in medical images, by improving the features to gain essential characteristics from the image for better diagnosis. ${ }^{[32]}$ The proposed method starts with a median filter and an unsharp mask filter to eliminate noise and sharpen the images. ${ }^{[32]}$ "Contrast Limited Adaptive Histogram Equalization and Average Filter" is used on the image for better results. Ibrahim et al. recommend a new method which is an extension to $\mathrm{HE}$, known as dynamic histogram equalization ${ }^{[19,33]}$ by preserving brightness. Here, the mean intensity of the resultant image is maintained almost equal to the input mean intensity of the image, thus maintaining the mean brightness in the image. In this, the input histogram is smoothened with a Gaussian filter, and then smoothened histogram is partitioned based on its local maximums. This creates a new dynamic range. Now, the histogram equalization process is applied separately to these partitions and maintains 
the mean input brightness based on the dynamic range. ${ }^{[18,19,33]}$

Arriaga-Garcia et al. present a modified approach to brightness-preserving dynamic histogram equalization. ${ }^{[18,19]}$ Over enhancement and excess brightness of histogram equalization shift the mean brightness and destroy the image appearance. ${ }^{[18,33]}$ "Bi-histogram equalization with adaptive sigmoidal functions" overcomes this by splitting the image histogram into a pair of sub-histograms with sigmoidal functions. ${ }^{[18,33]}$ It maintains the average brightness of the output image equal to the mean intensity of the input image. Based on the literature on bi-histogram equalization, ${ }^{[18]}$ an idea was proposed using the algorithm "Bi-Histogram Equalization with adaptive sigmoidal function" combines with the Sobel operator (horizontal and vertical) algorithm. This algorithm highlights the edges of both the nucleus and cytoplasm of the cell and reduces the unwanted enhancement and brightness caused by histogram equalization. ${ }^{[18,33]}$

Li et al. use a gray entropy value as the parameter to quantify the level of the pixel. Fuzzy mapping raises the capability to judge edge pixels. ${ }^{[34]}$ But it enhances the noise as well as improves the overall quality of the image. Choi et al. demonstrate three different fuzzy rule-based filtering systems. ${ }^{[23]}$ The filtering system is employed as the first part of removing impulse noise. Secondly, when images are severely contaminated, it outperforms the other methods with RMS (Root Mean Square) criterion and is used for smoothing noise. A third filtering system is used for noise attenuation. As a part of our study, Fuzzy Image Enhancement is combined with BiHistogram Equalization and observed the quality of enhancement. ${ }^{[23,34]}$ Imprecise boundaries and intensities are dealt with in the Fuzzy set theory. By making dark pixels darker and bright pixels brighter, better image enhancement is achieved using this new approach. ${ }^{[23,34]}$

In the Genetic algorithm, a simple chromosome structure is identified and used to find the finest gray pixels to enhance the image. ${ }^{[25,35]}$ The algorithm consists of four steps, such population initialization, applying fitness function, selection algorithm, and crossover and mutation operator. ${ }^{[25,35]}$ Hashemi et al. propose an algorithm based on an efficient contrast enhancement method. In the work, the fitness value for each chromosome is identified by the complete intensity of the image and the number of edges in an image. The fitness function is calculated by multiplying the number of edges calculated using the Sobel or canny operator and that is multiplied with the summation of the intensity values of the enhanced image. The proposed genetic method performs well with respect to contrast and detail enhancement of the resultant image. ${ }^{[24,25,35]}$

Ghaheri et al. present a review article on the application of the genetic algorithm in medicine. ${ }^{[25,35]}$ Applications of genetic algorithms in disease screening, diagnosis, treatment planning, prognosis, and health care management are discussed by the author. The study also suggests the importance of interdisciplinary courses, research activities, and the importance of engineering research in healthcare environments, to obtain new solutions and ideas for medicalrelated problems. In a recent study on genetic algorithm for image enhancement, Prabha et al. use the genetic algorithm and Fuzzy intensification operator to propose an efficient contrast enhancement algorithm. ${ }^{\left[{ }^{36]}\right.}$ The focus of the algorithm is to improve the visual details by manipulating the intensity information. From the result inferred, the Fuzzy logic and genetic algorithm-based method portray good performance. As per the literature, an enhancement algorithm is proposed by combining a genetic algorithm with bi-histogram equalization. ${ }^{[25,35,36]}$ In this approach, a simple chromosomal structure is defined, and used to find the appropriate gray level for enhancement. A sorted array of chromosome structures with random integers of size ' $n$ ' has been considered as the initial population. The count of edges and their overall intensity are used to calculate the fitness value for each chromosome. ${ }^{[25,35,36]}$ Based on the obtained fitness value, the best individual is selected and passed on to the next generation. To ensure smooth edges and to eliminate unwanted protrusion, the opening operation is iterated on the image. ${ }^{[17,35,36]}$

\subsection{Image segmentation}

Effective image segmentation and its information are very critical for decision-making on the severity of the disease. Localization and identification of the region of interest have been considered as meaningful input for further processing such as feature extraction and classification of disease. The improper segmentation of images results in over-segmentation or under-segmentation of the region of interest and leads to unreliable computer-aided diagnostic screening systems. As most of the abnormalities for malignancy detection are connected to the morphological features of cervical cells, accurate segmentation of features is essential for efficient screening. The main purpose of the process is to partition a digitally converted area into numerous regions, and the required region of interest (ROI) is extracted. In pap smear, cervical cell images, background, cytoplasm, and nucleus are considered as the three main regions. Depending upon the problem, appropriate regions can be segmented and their feature information can be used for classification purposes. The success and failure of the region of interest extraction greatly influence the classification process and disease diagnosis of smear images.

Chankong et al. use the Herlev dataset and segmented nucleus and cytoplasmic region using patch-based Fuzzy $\mathrm{C}$ mean clustering to segment cell features. ${ }^{[37]}$ Here single cell image is converted to grayscale and applied the median filter to smoothen and eliminate noise in the image and each patch of pixel cluster is represented by its middle value. ${ }^{[38]}$ By setting the threshold for the nucleus, a patch darker than the nucleus threshold is labeled as the nucleus which is implemented by Chankong et al., shown in Fig. 9. An accuracy of $99.2 \%$ for two-class and $93.7 \%$ for seven-class problems. ${ }^{[37]}$

Saha et al. studied to segment the nucleus from the overlapped images. The circular shape function (CSF) 

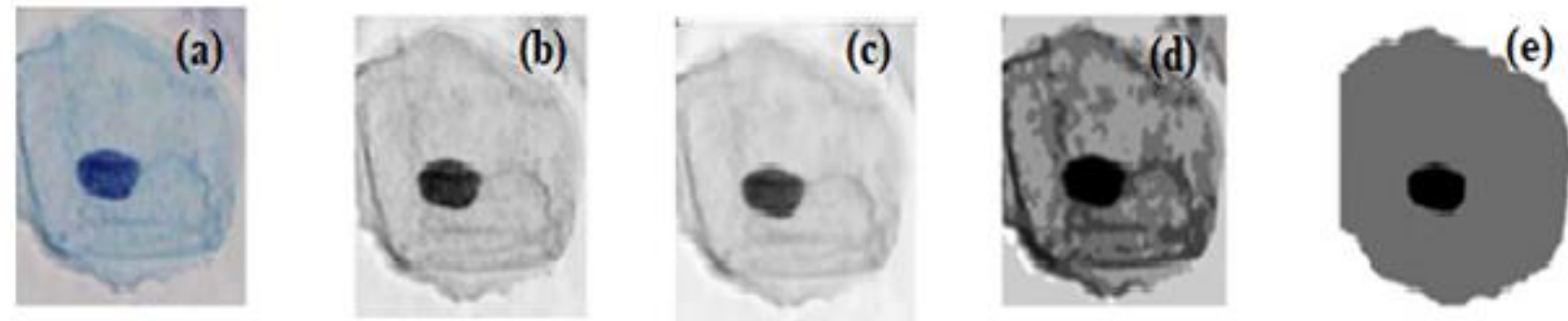

Fig. 9 Segmentation using patch-based Fuzzy C Means Clustering of Pap Smear Cervical Mild Cell Image of Herlev Dataset (a) Original Color Image, (b) Grayscale Image, (c) Median Filtered Image, (d) Patch Image after FCM and (e) After Patch Combining. Reproduced with permission from ${ }^{[37]}$.

technique ${ }^{[39]}$ is adopted to increase the performance of nucleus segmentation using the fuzzy c-means clustering algorithm. ${ }^{[35,39]}$ Due to the overlapping of cells, defining a global threshold value to binarize the image is a challenging task. An image histogram is used to identify the peaks and used to binarize the images into foreground and background. The proposed method attains an overall precision and recalls the value of $97 \%$ and $92 \% .{ }^{[35,39]}$

Wienert et al. propose a "contour-based minimum model cell detection and segmentation" approach to detect edges without depending on their shape properties. ${ }^{[40-44]}$ The proposed cell nuclei segmentation composes of six processing steps. The initial step starts with the detection of contours, evaluation, generation of non-overlapping segmentation, optimization, concave object separation followed by cell nucleus separation and other objects. ${ }^{[45]}$ This new model mitigates the morphological bias in segmentation and achieves a segmentation accuracy with $90 \%$ of precision and $81 \%$ of recall values. ${ }^{[40]}$

Tang et al. project a model using the "Fuzzy C Means (FCM)" method to quantify the chromatin patterns of cervical cells. ${ }^{\left[{ }^{30,35]}\right.}$ The study's purpose is to analyze granular chromatin distribution and hence to build a model. To detect the chromatin region, the area of chromatin, the distance between the two nearest chromatin pairs, and the eccentricity of chromatin are considered. The predefined number of clusters are used to segment the chromatin patterns and achieved different sensitivity level with acceptable accuracy. ${ }^{[30,35]}$

Talukdar et al. propose a method to segment color images. In this, the author initiates the clustering process by generating random numbers for each of the red, green, and blue channels. ${ }^{[41]}$ The membership value of generated random numbers is compared with the center of the cluster and the nucleus, cytoplasm, and background region are classified. Cluster validity measures such as partition coefficient, partition entropy, compactness, and separation validity function are used for cell segmentation. To measure the closeness of all input samples, partition coefficients are used. The compactness and separation validity functions are identified by taking the ratio of the average distance to their corresponding cluster centers and the minimum distance between cluster centers. ${ }^{[41]}$

Segmentation of the region of the nucleus of cell images is done using the "Fuzzy C Means" clustering algorithm ${ }^{[37-41]}$ because it can cluster data with uncertainty. The most fundamental issue in the bio-medical area is image segmentation. The active contour model has received a good amount of attention in medical imaging as it specifies a clear curve for the objects. As edge-based models show limitations in segmenting blur and weak edges, especially in medical images, most of the work concentrates on region-based models The region-based active contour model ${ }^{[42,43]}$ uses quantitative measures in and around the contour to control the progress of the curve. Chan Vese's model ${ }^{[41-43]}$ shows good results for appropriate segmentation of cell images as cervical cells possess homogeneous nature of pixel intensity distribution. Computational time is reduced by keeping the initial contour near the object boundaries.

Liu et al. work on a hybrid model that utilizes the region information of an image. ${ }^{[4]]}$ The authors' have combined the local and global information to achieve a better result for an image with intensity inhomogeneity. In this, the model uses the global mean intensities of the interior and exterior regions of images as per the $\mathrm{CV}\left(\mathrm{Chan}\right.$ Vese) model..$^{[43,44]}$ Computationally expensive and erroneous segmentation has been avoided by considering the weights between local and global fitting terms. ${ }^{[44]}$ Pratondo et al. propose an integrating model with region-based active contour and other algorithms such as "Support Vector Machines (SVM) and K Nearest Neighborhood (KNN)" for segmentation. ${ }^{[43,45]}$ The framework proposed by the authors utilizes probability scores which are normalized using a non-linear mapping. The Jaccard index (JI) and the Dice coefficient are used to evaluate the performance of segmentation. ${ }^{[43,45]}$

Aung et al. propose a region-based method using a levelset approach. ${ }^{[44,46]}$ This approach is performed based on two steps, such as curve evolution and segmentation process. The level set method aids in curve evolution, and segmentation of the region of interest has been performed using an active contour model. ${ }^{[46]}$ The model experimented with pap smear cervical cell images and gave acceptable accuracy for the segmented nucleus images. Saini et al. make a comparative study of edge-based and region-based active contour models. ${ }^{[47]}$ The intensification of malignancy in cervical cell images results in the irregular shape and size of the nucleus with blur edges. In region-based, ${ }^{[48]}$ image statistics of the 
foreground and background of the contours were used to control the evolution of the curve. This results in better performance of segmentation for weaker and blur-edged images. Edge-based model ${ }^{[48]}$ relies on the gradient of the image. The energy function of the evolving curve passes through true boundaries with weak and blur edges, resulting in the inaccuracy of the region of interest. ${ }^{[47,48]}$

"Chan Vese" model works well for images with homogeneous intensity and behaves differently for images with intensity inhomogeneity. ${ }^{[42,43]}$ The working of stochastic search method is based on the behavior of bird flocking. Particle swarm optimization algorithm, ${ }^{[48,49]}$ a stochastic search method, is applied to many optimization problems in the image analysis field, including enhancement, segmentation, and even in classification.

Segmentation of an image is to partition an image into a homogeneous region that contains similar pixels based on some features such as color, intensity, or texture. In cervical cell images, for segmenting the nucleus region which has homogeneous dark intensity pixels, Particle Swarm Optimization (PSO) is used to achieve good segmentation results. Mohsen et al. propose a multiple-level threshold image segmentation using the "Particle Swarm Optimization" algorithm. ${ }^{[25,48]}$ Depending upon the fitness function the algorithm gives the optimal threshold value to segment the region of connected pixels with specific gray-level intensity and achieves a good segmentation result. A hybrid model by Sukumar et al. uses Particle Swarm Optimization combined with an algorithm called "Extreme Learning Machine (ELM)" (PSO-ELM) which randomly decides concealed nodes and decides the output weights logically and achieves $96 \%$ segmentation accuracy. ${ }^{[49,50]}$

$\mathrm{S}$ Gautam et al. introduce a method using CNN (Convolutional Neural Network) which consists of selective pre-processing followed by patch-based CNN classification and post-processing methods. ${ }^{[51]}$ The algorithm is tested on the Herlev dataset ${ }^{[13]}$ and gives an accuracy of $92 \%$. Zhao et al. propose a new "Selective-Edge-Enhancement-based Nuclei Segmentation method (SEENS)" "52] in which selective search is combined with mathematical operators to segment whole slide cervical images into small regions of interest (ROI). To extract edge information, canny operator and mathematical morphology are used to achieve precision and recall values of $96 \%$ and $94 \%$, respectively. ${ }^{[52]}$

Huang et al. utilize a segmentation method based on a "multi-scale fuzzy clustering algorithm" that segments cervical cell clump images at different scales. ${ }^{[53]}$ Firstly, the algorithm is used to divide the cell clusters at different scales. Then a hierarchical tree is constructed based on the inclusion relationship between the segments. ${ }^{[53,54]}$ In the final phase, interesting nodes in the hierarchical tree are identified according to the proposed interesting degree based on the area prior. Wang et al. propose an algorithm based on depth information. The authors use a "tree domain structure and screening algorithm" based on a depth-first search according to the annular clustering characteristics of nucleus depth information in cervical cytology images. ${ }^{[55]}$ The candidate nucleus regions are finely segmented with an iterative level set algorithm based on adaptive radius morphological dilation. Positive predictive value and negative predictive value are calculated to assess the performance of segmentation and the results obtained are $99 \%$ and $95 \%$, respectively. ${ }^{[55]}$

\subsection{Feature extraction}

Cancer diagnosis mainly depends on the evidence obtained from the malignancy noticed in the cell structure and cell distribution. As the disease progresses, the morphological features, topology, texture, and color of cell features drastically vary and these can be considered as features for the classification of the disease. Extraction of appropriate features helps in converting an image into an understandable format. The appearance, structure, and distribution of pixel values within the image play a vital role in feature analysis. Clinical images possess an ample amount of texture information for disease diagnosis and clinical practice. The textural approach has been incorporated into the classification of pathological tissues and cells from the kidney, thyroid, liver, brain, cervix, lung, and many other organs. The co-occurrence matrix is one of the widely used texture analysis methods that aid in extracting statistical information such as the distribution of pairs of pixels in an image. Features extracted from pap smear images are categorized based on size, shape, topology, color intensity, texture, and CNN-based deep features. ${ }^{[56]}$

\subsubsection{Morphological and color features}

The distortion and increase in the size of the nucleus with respect to cytoplasm show the abnormality in the cells. Similarly, circularity and oval shape belong to a normal nucleus and abnormality portrays the irregular and coarse nature of cell structure and appearance. Size is expressed in terms of the radius, area, and perimeter of the cell whereas shape is in terms of symmetry, circularity, and length of the major and minor axis of the cell. ${ }^{[50,57]}$ Major and minor axis length shows the roundness of the nucleus and cytoplasm. Techniques such as thresholding, clustering, fuzzy, wavelet, and statistical techniques are used to extract the size and shape features of cytological images. Ripplet descriptors are used by Bora et al. to quantify color features. Color features mainly contribute to identifying the hyperchromatic and cytoplasmic changes. ${ }^{[58]}$ Before applying RT (Radon transform) decomposition, RGB (Red, Green, Blue) images are transformed to $\mathrm{YCbCr}$ color space to ensure that the textural characterizations of the images are independent of the color. ${ }^{[58]}$ As abnormal cell has darker color intensity composition when compared to normal cells, distinguishable patterns can be analyzed using a color intensity histogram. ${ }^{[57,58]}$ Recently, deep CNN plays a vital role in feature extraction in which color as well as other related image features are extracted automatically. ${ }^{[57,55]}$ 

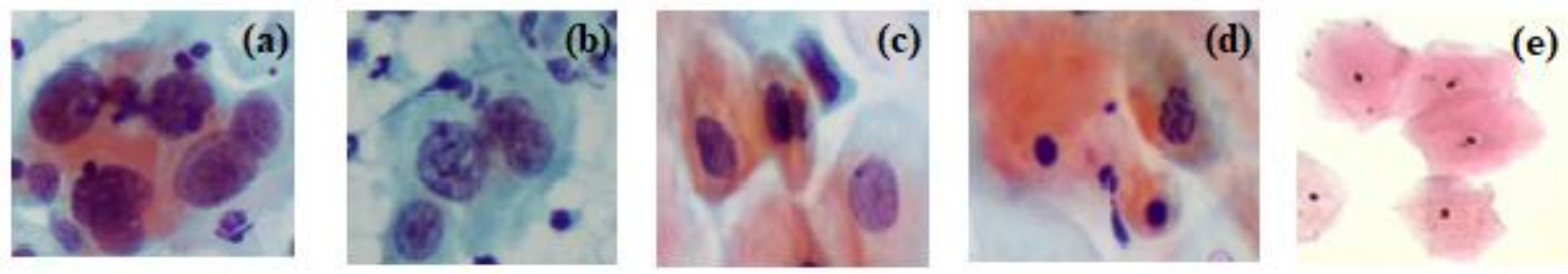

Fig. 10 Variation of chromatin distribution in cropped Pap smear Cervix Cell Images (a) Carcinoma Cell, (b) Severe Cell, (c) Moderate Cell, (d) Mild Cell, and (e) Normal Cell. Reproduced with permission from Kasturba Medical College (KMC), Department of Pathology, Manipal, India. (IEC 472/2013).

\subsubsection{Texture-based features}

The term hyperchromasia is a nuclear abnormality that occurs due to an increase in the chromatin content of the nuclei. Changes in the size and shape of the cell nucleus and its chromatin pattern is an important features or bio-marker for the identification of stages in disease progression. The threadlike chromatin structure inside the nucleus that carries genetic information replicates and shows drastic variations in the distribution of chromatin content as the disease progresses from normal to carcinoma which is presented in Fig. 10.

Authors ${ }^{[59]}$ use liquid crystal textures to investigate the phase transition temperatures of liquid crystals. ${ }^{[60]}$ In this method, texture properties such as "contrast, energy, homogeneity, and correlation" are computed, which are considered second-order statistical parameters. Textural features are identified on statistical measures that summarize the relative frequency distribution. The authors use textural features of three different kinds of image data in the category identification task. ${ }^{[61]}$ They achieve $89 \%$ of identification accuracy for "photomicrographs", $82 \%$ for "aerial photographic imagery", and "satellite imagery" of $89 \%$. Abnormality analysis is performed on 946 images. Normal cells hold a homogeneous texture and the frequency distribution of gray levels. In abnormal cells, cell replication results in uneven distribution of gray level frequency. This textural change is used to identify abnormal cells and disease diagnosis. In this paper, malignancy in the cervical cells is examined based on the texture and the occurrence of hyperchromasia, which are considered the two important morphological features that aid in distinguishing normal and abnormal cervical cells. ${ }^{[62]}$

Four stages of cervical screening techniques are reviewed. ${ }^{[63]}$ The authors achieve better accuracy on cytology and electromagnetic spectra data. They suggest the usage of the proposed intelligent system for cervical precancerous diagnosis for good classification performance. Texture parameters are one of the useful ways to get enormous information obtainable from medical images.

Castellano et al. discuss the importance of texture analysis with appropriate examples and techniques. ${ }^{[64]}$ The authors present a method to detect textile defects using texture analysis and neural networks. A feature extractor is defined based on the "Gray Level Co-occurrence Matrix (GLCM)," and textile defects are identified using neural network classifiers. The numerical simulation shows $100 \%$ accuracy for training and $100 \%$ for best and $91 \%$ worst testing and a comparison of two texture analyses has been discussed. ${ }^{[60]}$

"Markov Random Fields (MRF)" and "Gray Level Cooccurrence Probabilities" are used for the study of segmenting remotely sensed imagery and the GLCPs are more sensitive to texture boundary with good discrimination ability relative to Markov Random Fields (MRF). "Energy, Contrast, Variance, Correlation, Entropy, and Inverse Difference Moment" are some of the textural parameters considered for the work. Among that, Energy and Contrast parameters are found to be more efficient for discriminating different textural patterns. ${ }^{[65]}$ The authors work on statistical texture analysis of 2D images and present an overview of the methodologies and algorithms used for the work. Statistical approaches, such as basic, structural, and spectral, are used to describe the texture of the region. ${ }^{[57]}$ The basic approach yields smooth, coarse, and grainy characterizations of textures, and the structure deals with the arrangement of image primitives.

Spectral approaches aid in detecting global periodicity in an image resulting in the identification of high energy and narrow peaks in the spectrum. The authors ${ }^{[66]}$ investigate a new set of textural parameters extracted from the co-occurrence matrix. ${ }^{[59]}$ These parameters are used to train a "Support Vector Machine (SVM)" and ensembles. This novel extraction method improves the performance of standard methods. The authors propose an outline for the analysis of texture in medical images based on Vector Quantization (VQ). ${ }^{[67-69]} \mathrm{K}$ nearest neighbor classification, ${ }^{[67,68]}$ method is used to classify texture patterns and obtains an accuracy of $96 \%$. Authors work on textural color fusion in terms of color fidelity on different models. ${ }^{[70]}$ Computational models, like gray-to-color, color-to-color, and dichromatic-based models are used for this study. Certain textile fabrics are used as samples for this study. The similarity ratio of the image for each method is found to improve with texture strength applied for the study. ${ }^{[70]}$

\subsubsection{Deep CNN feature}

CNN-based deep learning techniques improve the image of the based computations by learning necessary features automatically from the input image. The performance of the traditional methods highly depends on the effect of handcrafted segmentation and another feature engineering. ${ }^{[70,71]}$ Cheng et al. perform whole slide image analysis for cervical 
cancer using deep learning and employed diverse deep techniques to improve the robustness of feature representation. Multilayer neural network perception mechanism aids in learning more abstract features and addresses several issues faced in conventional computer-aided diagnosing systems. ${ }^{\text {[70,71] }}$

Chandran et al. develop a computer-aided cervical cancer detection using colposcopy images using a deep neural network. The proposed method by the authors extracts discriminative features using an ensemble approach without segmentation and feature engineering stages. Mouser et al. propose deep neural network techniques using a multilayer perceptron classifier for disease classification. ${ }^{[56,72]}$ Recently, the transfer learning method is used in which the feature extraction part of the previously trained network is maintained and the classification part only is retrained on different datasets. ${ }^{[56,72]}$ Rahaman et al. introduce a deep cervix and a deep learning-based framework to classify cells based on "hybrid deep feature fusion (HDFF) techniques" capable of capturing more potential features with improved classification performance. ${ }^{[73]}$

The deep learning method outperforms recognizing patterns and high-level semantic features in large volumes of data, extracting relationships between complex features in the images, and identifying characteristics that are difficult to be perceived by the human brain. ${ }^{[16,73]}$ Lia et al. propose a mode based on the" faster RCNN-FPN (Region Based CNNFeature Pyramid Network) architecture" consisting of image input, feature pyramid network for feature extraction, feature map generation with region proposal network (RPN), classifier, and bounding box regressor. The introduction of the deformable convolutional layer into the feature extraction pyramid network improves the scalability of the model by detecting deformed cells of various sizes. ${ }^{[16,73]}$

\subsection{Image classification}

Accurate classification of pap smear images acts as a vital requirement for reliable screening and diagnosis. Classification is a challenging task, especially in medical images, which have minute variations in shape, size, color, and texture that contribute more to disease diagnosis. The performance of the classifier and classification can improve by obtaining and identifying well-distinct features and suitable classification algorithms. The progress in the area of machine intelligence and automation makes the pap smear screening process faster by automatically categorizing the sample into normal and malignant. Reducing false negative and false positive cases is one of the major tasks of a good classification algorithm that enables accurate, reliable, and early diagnosis of disease. Even with the numerous efforts and attempts to develop a complete, reliable diagnosing system, limitations still persist in achieving zero percent false-negative and zero percent false-positive cases. The review of relevant literature highlights the wide usage of machine learning and deep learning algorithms in this field.

\subsubsection{Traditional machine learning algorithms}

Initial classification of cytological images of the cervix is based on reviewing research publications on traditional machine learning algorithms. Under these algorithms, "Support Vector Machine (SVM)" is one of the popular and widely followed methods in many areas for classification purposes, especially in medical applications. Other classification methods such as logistic regression, K-nearest neighborhood, decision tree, and some of the ensemble methods like random forest and Ada-boost are reviewed, and performance analysis has been conducted with the proposed model. Zhang et al. present a naive SVM-based screening method and achieve a robust detection of disease by using multispectral pap smear image classification. ${ }^{[74]}$ This new screening method is showing significant improvement in the pixel-level classification of cervical cells.

Chen et al. discuss a semiautomatic analysis system for nucleus segmentation and contour detection. ${ }^{[75]}$ They compute morphological and textural features to train SVM to classify and discriminate malignant cells from normal cells and achieved an accuracy of $96.12 \%$ and $98.61 \%$ for 4 and 2 cluster classifiers. Sneha et al. propose a study to investigate the features derived from MR images for disease diagnosis. ${ }^{[67]}$ Second-order statistical features are used to predict cervical cancer tumors. The authors use SVM for their classification study and achieve an accuracy of $87 \%$ in their proposed model

Mariarputham et al. present a texture-based classification system that classifies the smear images into seven different classes. ${ }^{[12]}$ SVM shows acceptable classification accuracy with precision for normal squamous at $97.38 \%$, intermediate squamous at $93.89 \%$, mild dysplasia at $87.33 \%$, severe dysplasia at $58.52 \%$, and carcinoma in situ at $84.72 \%$. Linear, quadratic, and Radial Basis Function (RBF) SVM kernels are used for classification. The results show that better performance is achieved through a linear kernel Support Vector Machine (SVM) classifier than any other classifier.

Devi et al. explore different types and methods of neural networks for disease diagnosis. ${ }^{[68]}$ The "multi-layered perceptron" is used as an ANN (Artificial Neural Network) architecture that performs detection at a faster rate. A feedforward network and knowledge-based neural network are used where the input images are mapped with the rules and extracting the features which are used for classification.

Athinarayanan et al. develop a multiclass cervical classification system using pap smear images. ${ }^{[76]}$ Enriched "rough set texton co-occurrence matrix" (ERSTCM) and enriched "microstructure descriptor "(CABS) and concatenated feature extraction method (CFE) are used. These extracted features are tested over a fuzzy logic-based hybrid kernel support vector machine (FL-HKSVM) classifier and evaluated statistical parameters such as sensitivity, specificity, and accuracy. The concatenated feature extraction method has given better results than the other two classifiers. Miao et al. and Plissiti et al. develop an automated cell selection method from smear samples. ${ }^{[77,78]}$ SVM kernel functions are used to 
map the input data vector into higher dimensional spaces. The "Support Vector Machine" classifier with radial basis function showed good performance. The "Receiver Operating Curve (ROC)" curve is used to illustrate the performance of the classifier and obtained $98 \%$ of accuracy.

Wei et al. work on an automated method for the detection of cancer. They combine the "Gray Level Co-occurrence Matrix (GLCM)" and lesion area for classification using the SVM method and obtain an accuracy of $90 \%{ }^{[79]}$ Taha et al. focus on the classification phase by proposing a novel approach for cervical cancer detection. They use a pre-trained convolution neural network with an SVM at the back end. ${ }^{[80]}$ This innovative approach is evaluated with the Herlev dataset and achieved good performance in classifying cervical cells in terms of precision, recall, and accuracy values. Khan et al. use ensemble-based classification techniques for disease classification. Random Forest, Extreme Gradient Boosting, and AdaBoos ${ }^{[81]}$ are used for the training process. Random forest improves performance by adding many trees to reduce overfitting. Gradient Boosting produces a strong classifier by combining weak classifiers. Using this, a strong iterative model is built to optimize the loss function. AdaBoost uses progressive learning by combining several weak classifiers to build a meta classifier. Here authors use adaptive sampling to assign high weights to the misclassified events. Misclassified samples are selected in the next iteration to train the model in a better way using weighted voting. XGBoost outperforms other models as it handles sparse data and implements several optimization and regularization technique. ${ }^{[81]}$

Chen et al. propose a "transfer learning based snapshot ensemble" (TLSE) method by integrating snapshot ensemble learning ${ }^{[82]}$ with transfer learning. This method is evaluated on the Herlev dataset ${ }^{[13]}$ and achieves good accuracy. Transfer learning-based snapshot ensemble method (TLSE) is proposed for the fine-grained cervical cells classification task on the Herlev dataset for 7 classes and achieves an accuracy of $65 \% .{ }^{[82]}$ The authors address the big gap between the two and seven class accuracy and advise using GAN (Generative Adversarial Networks) ${ }^{[83]}$ for increasing the number of samples.

Ijaz et al. propose a cervical cancer prediction model in which authors adopt outlier removal techniques such as DBSCAN and iForest ${ }^{[84]}$ to improve the accuracy. The prediction model initially removes outliers using densitybased spatial clustering of applications with noise and isolation forest to balance the dataset through synthetic minority over-sampling technique "(SMOTE) and SMOTE with Tomek link (SMOTETomek)". ${ }^{[84]}$ Finally, a random forest classifier is used to classify the cells and achieves an accuracy of $97.47 \%$ for cytology images. ${ }^{[84]}$

In this authors also introduce a mobile app that collects the user's risk factor data and sends it to "Representational State Transfer (REST API)" "[84] and stores it in a secure remote server. To store a large amount of data, authors use NoSQL MongoDB. ${ }^{[84]}$ This is a naïve technique adopted by authors in the prediction model and the prediction results will be shown in the mobile app. The architecture design for this model by the authors is shown in Fig. 11.

Jahan et al. work on eight different machine learning algorithms such as "Multilayer Perceptron (MLP), Random Forest and k-Nearest Neighbor, Decision Tree, Logistic Regression, Support vector clustering (SVC), Gradient Boosting, and AdaBoost" to predict cervical cancer. ${ }^{[55]}$ To identify the best features, a combination of feature selection techniques such as "Chi-square, Select Best, and Random Forest" are used. Among the classifiers, Multilayer Perceptron achieves the highest accuracy of $98 \%$ and decision tree achieves the least accuracy of $86 \%{ }^{[84]}$

Ali et al. propose a study on machine learning-based statistical analysis. The authors' split the dataset into four parts based on the class attributes such as "biopsy, cytology,

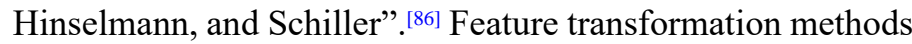
such as $\log$, sine function, and Z-score ${ }^{[86]}$ are applied to these datasets. Supervised machine learning algorithms like Random Tree (RT), Random Forest (RF), and Instance-Based $\mathrm{K}$-nearest neighbor (IBk) are used to assess the performance of classification. An RT algorithm provides the best classification accuracy for the biopsy (98.33\%) and cytology (98.65\%) data, whereas RF and IBk provide the best performance for Hinselmann (99.16\%), and Schiller $(98.58 \%))^{[86]}$ To interpret the results of various classifiers, statistical measures such as accuracy, sensitivity, specificity, precision, recall, F-Measure, AUROC, and kappa statistics are exploited. ${ }^{[86]}$

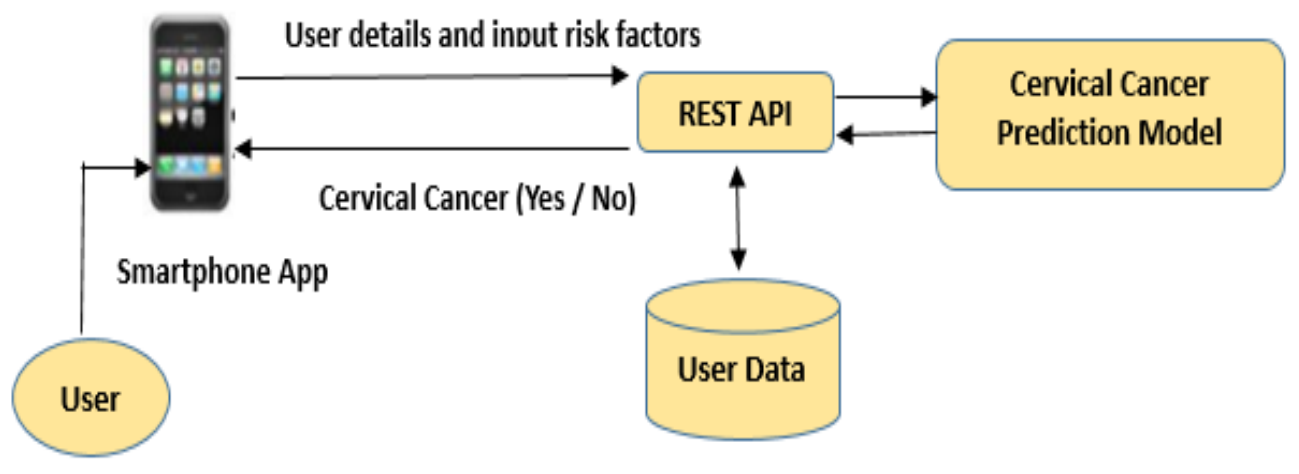

Fig. 11 Mobile App Cervical Cancer Prediction Model Architecture. Reproduced with permission from ${ }^{[84]}$. 


\subsubsection{Deep neural networks}

Timely cancer detection is a crucial task for health care. "Artificial Neural Network", ${ }^{[87]}$ inspired by the bio-nervous system, is used to solve a complex pattern that includes layered architecture with one or more hidden layers. ${ }^{[8,89]}$ Deep learning architecture, ${ }^{[87-89]}$ consists of numerous hidden layers and captures the non-linear relationship of the complex pattern, and makes wise predictions. Convolution neural network is one of the widely used deep neural network architectures ${ }^{[17,79]}$ that have useful applications in image recognition and analysis. ${ }^{[87-89]}$ It learns features at different levels of abstraction and makes a system to learn all potential features from basiclevel features such as arcs, lines, edges, and pixel intensity of an image to complex-level features such as objects and shapes without depending on features extracted by humans. ${ }^{[87-89]}$

Taha et al. classify cells using deep feature learning convolution neural network. ${ }^{[80]}$ They achieve good classification results and acquire $98.3 \%$ accuracy on the Herlev data set. Zhang et al. use a pre-trained CNN architecture to obtain the features, and these features are given as input to the SVM classifier and attained good classification results on the Herlev dataset. ${ }^{[89]}$ The article by Ravi et al. focuses on deep learning, particularly in bioinformatics, medical imaging, and public health. The authors make a comparative study of various deep learning architectures and outline the advantages in health-related areas. As this model allows the automatic generation of features, it aids in reducing human involvement in the feature extraction phase. ${ }^{90]}$

Based on the empirical evaluation and performance analysis, the Convolution Neural Network ${ }^{[26]}$ is suggested for high-performance computer-aided detection, especially in the area of medical imaging. ${ }^{[1]}$ Authors propose a digital-based diagnosis deep learning technique using a Convolution Neural Network $(\mathrm{CNN})^{[92]}$ for the classification of interstitial lung diseases and achieve $85.5 \%$. Hyeon et al. train a model using a CNN ${ }^{[93]}$ and extract feature vectors of images. These features are trained using the SVM classifier and acquire a performance of $78 \% .^{[93]}$

Different types of architecture in Artificial Neural Networks (ANN) ${ }^{[68]}$ such as "multi-layered perceptron, back propagation neural network, radial basis function network, fuzzy RBF network, Convolution neural network, and feedforward network" are analyzed. ${ }^{[68]}$ Mundhra et al. study the peripheral blood smear analysis system ${ }^{[87,94]}$ and employ deep models to analyze blood smears for localization and classification. The authors achieve $98 \%$ and $91 \%$ of specificity and sensitivity, respectively. ${ }^{[94]}$

Rehman et al. conduct experiments on malignant and benign cell assessment on the Herlev dataset. Achieving good classification accuracy mainly depends on a large amount of labeled data and it's a challenging task in medical diagnosis. ${ }^{[95]}$ So, transfer learning is adopted which aids in reference to overfitting and hyperparameter-related issues. After extracting the features using a convolutional neural network, classification is performed using three different classifiers such as. Gentle Boost Ensemble of Decision Tree, Softmax Regression and SVM achieve good accuracy using the ensemble method. ${ }^{[95]}$ The CNN architecture used by the authors is shown in Fig. 12.

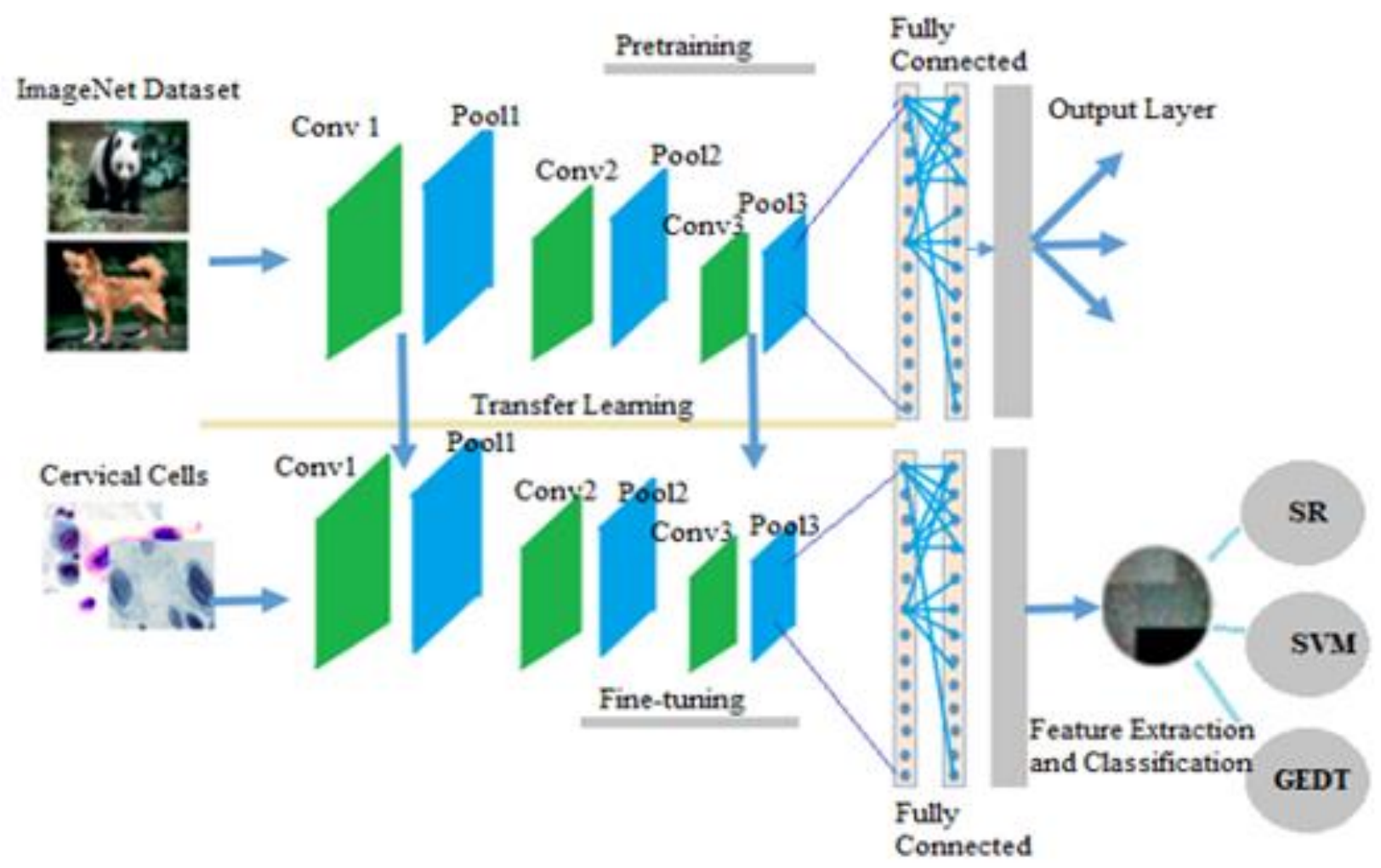

Fig. 12 Feature learning and classification using convolutional neural network. Reproduced with permission from ${ }^{\text {[95] }}$ 
Li et al. propose a novel framework based on "Faster RCNN-FPN architecture" ${ }^{\text {[16] }}$ for the detection of abnormal cervical cells. This model can be used as an AI tool for the automated detection of cervical cancer cells in whole slide images of pap smears. ${ }^{\left[{ }^{16]}\right.}$ Here authors introduce a deformable convolution layer into the feature extraction pyramid network resulting in the accurate detection of deformed cells of various sizes. Spatial context information also plays a very important role in the detection of abnormal cells. ${ }^{[16]}$

Diniz et al. work on deep ensemble methods in pap smear image classification. The objective of the model is to maximize the number of true positive results and to minimize the number of false negative results. Authors have used convolutional neural networks and EfficientNet networks ${ }^{[83]}$ which are state-of-the-art architectures for ImageNet dataset classification. The main block of architecture considered in the work includes the MobileNet block, InceptionNet block, and EfficientNet block. ${ }^{[83]}$ For classification purposes, $80 \%$ of the images are used to train the networks and $20 \%$ for testing, and $20 \%$ of the images are considered from the training set for validation. The proposed ensemble model with these architectures used for the study portrays an accuracy of $97 \%$ in cervical cell classification. Mohammed et al. work on pretrained deep neural network architecture. ${ }^{[96]}$ A publicly available dataset, named SIPaKMeD is used in which a total number of 4049 single-cell images are manually cropped from 966 full-slide Pap smear images. ${ }^{[96]}$ Among the different pretrained classifiers used for the study, DenseNet169 outperforms with an average accuracy, precision, recall, and F1-score of $0.990,0.974,0.974$, and 0.974 , respectively. ${ }^{[96]}$

\section{Evaluation metrics in cervical cancer diagnosis}

Various evaluation metrics are adopted by different authors to elucidate the performance of the classification model. For most of the medical image classification, the effectiveness of the model is tested using a confusion matrix and receiver operating characteristic analysis. The distinct metrics used for the analysis include Sensitivity, Specificity, Accuracy, and F1 score. ROC ${ }^{[16]}$ (Receiver Operating Characteristics) is obtained by plotting the true positive rate against the false positive rate. ${ }^{[97,98]}$ The prediction output consists of four outcomes such as true positive (positive samples correctly classified as positive), true negative (negative samples correctly classified as negative), false positive (the number of negative samples incorrectly classified as positive ), and false negative (the number of positive samples incorrectly classified as negative). ${ }^{[97,98]} \mathrm{F} 1$ score is the measure to find the balance between precision and recall and is used to identify the uneven class distribution. Table 3 shows the different performance metrics used to assess the model.

Precision also called a positive predicted value is the possibility that a person will have the disease after receiving a positive test result. ${ }^{[17,72,83]}$ Recall or sensitivity is the percentage of people who test positive out of all those who have the disease. Specificity is the proportion of people who test negative among all those who do not have the disease. ${ }^{[17,72,83]}$ Accuracy is the measure of correctly expected observation to the total observations. ${ }^{[17,72,83]} \mathrm{F} 1$ score is the weighted average of precision and recall which conveys the balance between both. ROC curve has been obtained by plotting TPR (True Positive Rate), which is the probability of predicting true positive as positive against TNR (True Negative Rate), which is the probability of predicting true negative as negative. The area under the curve (AUC) indicates the accuracy of the mode. ${ }^{[17,72,83]}$

Table 3. Evaluation Metrics for the Classification Model.

\begin{tabular}{|c|c|}
\hline Performance Metric & Calculation Formula \\
\hline Precision $^{[72-83]}$ & $\mathrm{TP} /(\mathrm{TP}+\mathrm{FP})^{[72-83]}$ \\
\hline Recall (Sensitivity) ${ }^{[72-83]}$ & $\mathrm{TP} /(\mathrm{TP}+\mathrm{FN})^{[72-83]}$ \\
\hline Specificity ${ }^{[72-83]}$ & $\mathrm{TN} /(\mathrm{TN}+\mathrm{FP})^{[72-83]}$ \\
\hline Accuracy ${ }^{[72-83]}$ & $(\mathrm{TP}+\mathrm{TN}) /(\mathrm{TP}+\mathrm{TN}+\mathrm{FP}+\mathrm{FN})^{[72-83]}$ \\
\hline F1 Score ${ }^{[72-83]}$ & $\begin{array}{c}2 \times((\text { Precision } \times \text { Recall }) /(\text { Precision }+ \\
\text { Recall }))^{[72-83]}\end{array}$ \\
\hline AUROC $^{[72-83]}$ & $\begin{aligned} \mathrm{TPR} & =\mathrm{TP} /(\mathrm{TP}+\mathrm{FN})^{[72-83]} \\
\mathrm{TNR} & =\mathrm{TN} /(\mathrm{TN}+\mathrm{FP})^{[72-83]}\end{aligned}$ \\
\hline
\end{tabular}

Rehman et al., exhibit the performance of the classification model using above mentioned performance metrics for automated screening of cervical cancer using a convolutional neural network. ${ }^{[95]}$ Chandran et al. use an ensemble-based deep learning network and the same evaluation metrics to find the performance of the model. ${ }^{[20,72]} \mathrm{Li}$ et al. apart from the metrics outlined above, ROC is used to detect the performance of the model which exhibits the relation between true positive and false positive rates. ${ }^{[16]}$ Ijaz et al. work on removing outliers using outlier detection techniques such as DBSCAN and iForest. ${ }^{[84]}$ Here, the authors use precision, sensitivity, specificity, F1 score, and accuracy for identifying the performance of the model. As the confusion matrix is the common evaluation method used to solve classification problems, Jahan et al. and Diniz et al. use the same evaluation measures outlined above for automated invasive cervical cancer detection. Here multilayer perceptron model used by Jahan et al. achieves the highest accuracy of $98 \% 0^{[83,85]}$ Razali et al. use the same evaluation metrics mentioned above to evaluate the performance of classification techniques such as "Naive Bayes, Decision Tree, k-Nearest Neighbors, Sequential Minimal Optimization, Random Forest Decision Tree, Multilayer Perceptron, Neural Network, and Simple Logistic Regression". ${ }^{[99]}$ Mohammed et al. use several supervised machine learning algorithms to evaluate the performance of diagnosis in the early stage. Apart from other statistical metrics outlined above, the author use kappa statistics which is used to test interrater reliability to represent the data that is collected for the study are the correct representation of variables measured. Authors achieve the best kappa statistics value for biopsy images at $97 \%$ and for cytology images at $87 \%{ }^{[96]}$ 
Table 4. Summary of highlighted works on cervical cell segmentation and classification.

\begin{tabular}{|c|c|c|c|c|}
\hline Author's Name & Datasets Used & Segmentation & Classification & Accuracy Obtained \\
\hline $\begin{array}{l}\text { Athinarayanan et } \\
\text { al. (2017) }\end{array}$ & $\begin{array}{l}\text { Images were collected from } \\
\text { Muthamil Hospital, Tamilnadu, } \\
\text { (952 Images) }\end{array}$ & Not mentioned & 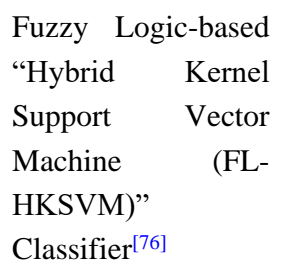 & 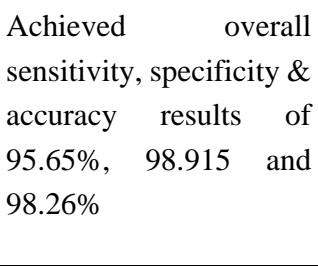 \\
\hline $\begin{array}{l}\text { Athinarayanan } \\
\text { and Srinath } \\
(2016)\end{array}$ & Herlev Dataset & Region props algorithm & $\begin{array}{lr}\text { RBF and kernel- } \\
\text { based } \\
\text { classification } \\
\text { used. }\end{array}$ & $\begin{array}{l}\text { Achieved an accuracy of } \\
94 \%\end{array}$ \\
\hline $\begin{array}{l}\text { Athinarayanan, } \\
\text { S., \& Srinath, M. } \\
\text { V. (2016) }\end{array}$ & Herlev Dataset & $\begin{array}{l}\text { Modified Otsu } \\
\text { Thresholding Algorithm }\end{array}$ & $\begin{array}{l}\text { Support Vector } \\
\text { Machine, KNN, and } \\
\text { ANN Classification } \\
\text { techniques }\end{array}$ & $\begin{array}{l}\text { SVM performed better } \\
\text { compared to KNN and } \\
\text { ANN with } 86 \% \text { of } \\
\text { accuracy }\end{array}$ \\
\hline Bora et al. (2016) & Herlev Dataset & $\begin{array}{l}\text { Used Deep features using } \\
\text { CNN without segmenting } \\
\text { the region of interest. }\end{array}$ & $\begin{array}{l}\text { CNN with the least } \\
\text { square version of } \\
\text { SVM is used with } \\
\text { softmax. }\end{array}$ & $\begin{array}{l}\text { Showed a good } \\
\text { accuracy for } 3 \text { classes } \\
\text { and } 2 \text { classes in the } \\
\text { range of } 90-95 \%\end{array}$ \\
\hline Bora et al (2017) & Herlev dataset & $\begin{array}{l}\text { The k-Mean color } \\
\text { segmentation approach }\end{array}$ & $\begin{array}{l}\text { Ensemble } \\
\text { classifier }^{[58]}\end{array}$ & $\begin{array}{l}\text { Achieved an accuracy of } \\
96.51 \% \text { ( } 2 \text { class }) \text { and } \\
91.71 \% \text { ( } 3 \text { class })\end{array}$ \\
\hline $\begin{array}{l}\text { Chankong et al. } \\
\text { (2014) }\end{array}$ & Herlev dataset & $\begin{array}{l}\text { Patch-based FCM clustering } \\
\text { method }\end{array}$ & FCM algorithm & $\begin{array}{l}\text { Accuracy of } 93.78 \% \text { and } \\
99.27 \% \text { for the } 7 \text {-class } \\
\text { and 2-class problems }\end{array}$ \\
\hline Chen et al. (2014) & $\begin{array}{l}1814 \\
\text { cell images were captured from } 42 \\
\text { Pap smears }\end{array}$ & $\begin{array}{l}\text { Normalized cross- } \\
\text { correlation (NCC) method. }\end{array}$ & $\begin{array}{l}\text { support vector } \\
\text { machine used to } \\
\text { classify four clusters } \\
\text { and } 2 \text { cluster }\end{array}$ & $\begin{array}{l}\text { Achieved an accuracy of } \\
96 \% \text { in } 4 \text { clusters and } \\
98 \% \text { in } 2 \text { cluster }\end{array}$ \\
\hline Devi et al. (2016) & $\begin{array}{l}\text { Collection of datasets with a } \\
\text { maximum of } 20 \text { samples to } 1400 \\
\text { sample images and used Pathology } \\
\text { Anatomy Images }{ }^{[68]}\end{array}$ & nil & $\begin{array}{l}\text { Multi-layer } \\
\text { perceptron, } \\
\text { cascaded and hybrid } \\
\text { MLP, and CNN } \\
\end{array}$ & $\begin{array}{l}\text { MLP architecture gives } \\
\text { better accurate results } \\
\text { with } 98 \% \text { of accuracy }\end{array}$ \\
\hline $\begin{array}{l}\text { Mariarputham et } \\
\text { al. (2015) }\end{array}$ & Herlev Dataset & $\begin{array}{l}\text { The geometric active } \\
\text { contours were used }\end{array}$ & \begin{tabular}{lr}
\multicolumn{2}{l}{ Used SVM } \\
classifier \\
using & selected \\
texture & image \\
feature &
\end{tabular} & 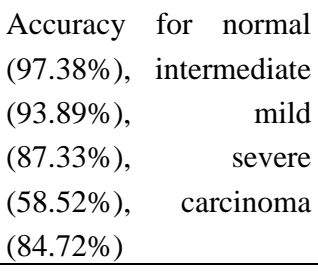 \\
\hline $\begin{array}{l}\text { Mundhra et al. } \\
\text { (2017) }\end{array}$ & nil & Deep learning models & $\begin{array}{l}\text { Used an ensemble of } \\
\text { deep-learning } \\
\text { models }\end{array}$ & $\begin{array}{l}\text { Achieved } 98 \% \text { of } \\
\text { specificity and } 91 \% \text { of } \\
\text { sensitivity for } \\
\text { identifying common cell } \\
\text { types }\end{array}$ \\
\hline $\begin{array}{l}\text { Mbaga et al. } \\
(2015)\end{array}$ & Herlev Dataset & $\begin{array}{l}\text { Used canny edge detector } \\
\text { algorithm for the edge } \\
\text { detection }\end{array}$ & $\begin{array}{l}\text { Used } \begin{array}{l}\text { SVM } \\
\text { Classifier with } \mathrm{RBF} \\
\text { and polynomial } \\
\text { kernels }\end{array} \\
\end{array}$ & $\begin{array}{l}\text { Achieved average } \\
\text { accuracy of } 92.961 \%, \\
\text { sensitivity } 90.833 \% \text { and } \\
\text { specificity } 80.39 \%{ }^{[89]}\end{array}$ \\
\hline $\begin{array}{l}\text { Sharma et al. } \\
(2016)\end{array}$ & Single cells datasets & $\begin{array}{l}\text { Used min-max and edge } \\
\text { detection methods }\end{array}$ & $\begin{array}{l}\text { K-nearest neighbor } \\
\text { method }\end{array}$ & Accuracy $82.9 \%$ \\
\hline
\end{tabular}


Continued

\begin{tabular}{|c|c|c|c|c|}
\hline Author's Name & Datasets Used & Segmentation & Classification & Accuracy Obtained \\
\hline $\begin{array}{l}\text { Sornapudi et al. } \\
\text { (2018) }\end{array}$ & 133 digitized histology images & $\begin{array}{l}\text { The used } \text { simple linear } \\
\text { iterative } \\
\text { algorithm }\end{array}$ & $\begin{array}{l}\text { Deep learning based } \\
\text { on nuclei } \\
\text { segmentation and } \\
\text { training with a } \\
\text { convolutional neural } \\
\text { network }\end{array}$ & $\begin{array}{l}\text { Achieved an overall } \\
\text { nuclei detection (object- } \\
\text { based) accuracy of } \\
95.97 \%\end{array}$ \\
\hline Taha et al. (2017) & Herlev Dataset & $\begin{array}{l}\text { Region-based and contour } \\
\text { based of method }\end{array}$ & $\begin{array}{l}\text { Used Deep learning } \\
\text { approach }\end{array}$ & $\begin{array}{l}\text { recall, } \\
\text { specificity, } \\
\text { accuracy with scores of } \\
99.51 \%, \\
97.67 \% \text {, and } 99.19 \%, \\
\text { respectively for } 2 \text { class } \\
\text { classification }\end{array}$ \\
\hline $\begin{array}{l}\text { Taha, B., Dias, J., } \\
\& \quad \text { Werghi, N. } \\
(2017)\end{array}$ & Herlev & $\begin{array}{l}\text { Region-based methods and } \\
\text { contour-based methods }\end{array}$ & $\begin{array}{l}\text { Alex Net is used as a } \\
\text { feature extractor and } \\
\text { then coupled with a } \\
\text { classic SVM } \\
\text { classifier }\end{array}$ & $\begin{array}{l}\text { Achieved recall, } \\
\text { precision, specificity, } \\
\text { and accuracy with } \\
\text { scores of } 99.51 \% \text {, } \\
99.5 \% \text {, 97.67\%, and } \\
99.19 \%\end{array}$ \\
\hline Wei et al. (2017) & cervical histological images used & $\mathrm{k}$-means clustering & SVM & $\begin{array}{l}\text { Achieved an accuracy of } \\
90 \% \text { in recognition of } \\
\text { cervical cancer }\end{array}$ \\
\hline $\begin{array}{l}\text { Zhang et al. } \\
(2017)\end{array}$ & Herlev dataset & $\begin{array}{lr}\text { Deep features, using } \\
\text { convolutional neural } \\
\text { networks (ConvNets) }\end{array}$ & Deep CNN & $\begin{array}{l}\text { Achieved accuracy } \\
(98.3 \%)\end{array}$ \\
\hline Wu et al., (2018) & H\&E-stained tissue section used & $\begin{array}{l}\text { Deep convolutional neural } \\
\text { networks (ConvNets) }\end{array}$ & Deep CNN & $\begin{array}{l}\text { Achieved an average } \\
\text { accuracy of }(93.3 \%)\end{array}$ \\
\hline $\begin{array}{l}\text { Shanthi et al., } \\
\text { (2019) }\end{array}$ & Herlev Dataset and KMC Dataset & $\begin{array}{l}\text { Deep convolutional neural } \\
\text { networks (ConvNets) }\end{array}$ & $\mathrm{CNN}$ & $\begin{array}{l}\text { For } 2,34 \text {, and } 5 \text { classes, } \\
99 \%, 94.8 \%, 96 \% \text {, and } \\
94.1 \% \text { classification } \\
\text { accuracy was achieved. }\end{array}$ \\
\hline $\begin{array}{l}\text { Chandran et al., } \\
\text { (2021) }\end{array}$ & $\begin{array}{l}\text { Detection of cervical cancer } \\
\text { using colposcopy images }\end{array}$ & Deep Features & $\begin{array}{l}\text { Used Ensemble } \\
\text { Deep Learning } \\
\text { Network }\end{array}$ & $\begin{array}{l}\text { Achieved high } \\
\text { sensitivity of } 92 \% \text { and } \\
\text { specificity of } 96 \%\end{array}$ \\
\hline $\begin{array}{l}\text { Mohammed et } \\
\text { al., (2021) }\end{array}$ & $\begin{array}{l}\text { Single-cell classification using } \\
\text { SIPaKMeD dataset }\end{array}$ & Deep Features & $\begin{array}{l}\text { Pre-trained deep } \\
\text { convolutional } \\
\text { neural network } \\
(\mathrm{DCNN}) \text { image } \\
\text { classifiers }\end{array}$ & $\begin{array}{l}\text { Achieved accuracy of } \\
99 \%\end{array}$ \\
\hline
\end{tabular}

\section{Classification performance}

The comparative study on the classification performance of smear images overviews the methods employed by various authors in their publications that focus on computer-assisted early diagnosis of disease. A detailed review of various classification approaches for cervical cells proposed in the literature is presented in Table $4{ }^{[96-107]}$ In this comparative study, the comparison is made between the SVM-based work as well as the deep CNN model for cell image classification.

The reported accuracy in the surveyed papers on classifications can be improved by incorporating novel methods in ROI enhancement, segmentation, feature extraction, selection, and classification. Plissiti et al. classify the cell into malignant and nonmalignant based on color intensity features. The relative nucleus area is used as a feature to identify the cancerous cells and achieves good differentiation between normal and cancerous cells, as cancer cells contain a larger distribution of darker pixels. ${ }^{[78]}$

Alqahtani et al. used the ANN classification model to forecast the happening of cancer in the prostate and compared the result with SVM, a Logistic regression model, and a Decision tree. ${ }^{[87]}$ ANN exhibits a good success rate of $99.36 \%$ 
where authors achieve $84.95 \%, 90.34 \%$, and $88.52 \%$ in logistic regression, decision tree classifier, and support vector machine. Mredhula et al. apply a model that involves two phases. In the first phase, the median filter is used to remove the noises. Pixel surge model (PSM) and "Probabilistic Principal Component Analysis" (PPCA) are used to improve the denoised image output with morphological operations, filtering, and region props methods. ${ }^{[100]}$ In the second phase, the neural network-based classifier is used for classification and shows the best performance with an accuracy of $88 \%{ }^{[100]}$

Devi et al. experiment with the classification model using an artificial neural network that produces good results. The authors perform a survey on different types of architecture such as Multi-Layer perceptron (MLP), cascaded and hybrid MLP, CNN, RBF network, feed-forward network, and knowledge-based network. Among all the architectures experimented on, MLP architecture gives better accurate results with $98 \%$ of accuracy. ${ }^{[68]}$ Chen et al. develop a model using morphological and texture features of the nucleus to train SVM classifiers to classify four and two-class clusters. They achieve an accuracy of $96 \%$ in 4 clusters and $98 \%$ in 2 clusters. ${ }^{[75]}$ Athinarayanan et al. use a "Fuzzy Logic-based Hybrid Kernel Support Vector Machine" (FL-HKSVM) Classifier ${ }^{[68,76]}$ and achieve overall sensitivity, specificity \& accuracy results of $95.65 \%, 98.91 \%$, and $98.26 \%{ }^{[76]}$ Taha et al. propose a deep approach, and the model outdoes performance in terms of recall, precision, specificity, and accuracy with scores of $99.51 \%, 99.5 \%, 97.67 \%$, and $99.19 \%$ respectively for 2 class classifications. ${ }^{[80]}$ Bora et al. worked on a model that reduces the computational demand by using the least square version of SVM (LSSVM) and used softmax for multiclass classification. The proposed method shows good accuracy for three classes and two classes in the range of $90-95 \% \cdot{ }^{[58]}$

Mariarputham et al. propose a model using SVM with textural features. The precision value achieved by the classifier is discussed for normal squamous (97.38\%), intermediate squamous $(93.89 \%)$, mild dysplasia $(87.33 \%)$, severe dysplasia $(58.52 \%)$, and carcinoma in situ $(84.72 \%)$. ${ }^{[12]}$ Athinarayanan et al. propose a multi-stage system for cancer diagnosis. Noise removal is performed and extracted textural features. Then classification is performed based on these extracted features. RBF and kernel-based SVM classification is used and achieved $94 \%$ by the classification. ${ }^{[101]}$

Wei et al. propose a model using SVM. Texture features are extracted, such as correlation, entropy, uniformity, and energy, and achieve an accuracy of $90 \%$ in the recognition of cervical cancer. ${ }^{[79]}$ Qayyum, Adnan, et al. review convolutional neural network-based techniques used for medical image analysis and computer-aided diagnosis. ${ }^{[102]}$ Comparison between machine learning and deep learning models by Suzuki, K. ${ }^{[103]}$ infers that without object segmentation or feature extraction, image data could be analyzed directly and considered as the essential difference between ML and deep learning. Sornapudi et al. propose deep learning (DL)-based nuclei segmentation ${ }^{[104]}$ and training with a convolutional neural network. The proposed approach is evaluated with an accuracy of $95.97 \%$. Mundhra et al. employ an ensemble of deep learning models ${ }^{[94]}$ to classify the red blood cells, white blood cells, and platelets along with their subtypes. The authors achieve $98 \%$ of specificity and $91 \%$ of sensitivity for identifying common cell types. ${ }^{[87,94]}$

Wieslander et al. use CNN to prove the accuracy of image classification tasks. The performance is evaluated for ResNet and Visual Geometry Group (VGG) deep CNN with an accuracy of $82-85 \%$. Ravi et al. present a brief review of deep learning in health science. The key application area such as bioinformatics, medical imaging, and medical informatics along with public health have been reviewed. ${ }^{[90]}$

Sarwar et al. present an ensemble method that combines the decision from the various models and is used for the study to improve the performance of classification. ${ }^{[105]}$ Shen et al. introduce deep learning methods and their contribution to image registration, cell structures detection, and tissue segmentation in computer-aided disease diagnosis or prognosis. ${ }^{[107]}$ Sharma et al. propose an idea of integrating segmentation methods with the region of interest locating methods to improve the segmentation and classification accuracy and achieve an accuracy of $93.7 \%$ with the Herlev dataset $^{[108]}$ Clausi et al. use Gray Level Co-occurrence Probabilities (GLCP) for segmenting images and concluded that the GLCPs are more sensitive to texture boundary which results in good classification accuracy. ${ }^{[109]}$ William et al. present a review on image analysis and machine learning techniques for automated cervical cancer screening and identified K-nearest-neighbors and support vector machines algorithms as an excellent classifiers for cervical images with an accuracy of over $99.27 \%$ and $98.5 \%$ respectively for 2-class classification problem. ${ }^{[110]}$ Plissiti et al. proposed a method, to examine the performance of unsupervised (fuzzy C-means) and supervised (support vector machines) classification techniques. Using these techniques, the results were very promising, even in the case of images with a high degree of cell overlapping. ${ }^{[111]}$ Deepak et al. perform classification using an automated image analyzer which effectively screens $60 \%$ of normal cells without any human intervention and suspicious smears are sent for further analysis. ${ }^{[112]}$

\section{Conclusion}

Pap smear cost-effective cancer screening plays a major role in reducing the mortality rate with proper evaluations at regular intervals. Incorporating intelligent algorithms in cancer diagnosis can contribute much to mass screening programs, especially in low- and middle-income countries. Insufficient awareness about the disease, low financial resources, weak health services, and poor access to treatment are some of the other reasons for the increased mortality rate of this cancer. To develop and validate the new computerassisted systems, a lot of research is required. Major drawback of existing screening systems includes cost-effectiveness, the 
accuracy of automation-assisted systems, and low sensitivity rates. The success of the computer-aided diagnostic system depends on the factors such as efficient image acquisition to obtain digitized images from smear slides, image enhancement to remove noise and other unwanted information and to enhance the region of interest, segmentation to extract the region of interest, and classification, which is one of the most important phases in the computer-assisted disease diagnosis to determine the cancerous and non-cancerous images.

Relevant literature has highlighted some of the techniques used for preprocessing, segmentation, feature extraction, and classification of pap smear images. From the literature review, it has been observed that ineffective preprocessing and poor segmentation leads to poor results in the classification phase. So efficient preprocessing and segmentation is a mandatory step prior to classification. This review provides an overview of the severity of the disease, its impact on world disease statistics, the need for an automated system, and current advancement in computerized analysis. A thorough introduction of domain knowledge and technical aspects with respect to existing and emerging computational methods addressing image preprocessing, enhancement of region of interest, segmentation of most appropriate features, hyperchromatic-based feature extraction, and various classification methods were presented.

The recent literature highlights the use of transfer learning that reduces the need of training the whole network over again. Leveraging the concept of deep CNN to this also enhances the model accuracy as the transfer learning phase makes the training process easier. This review emphasizes the usage of naïve techniques in outlier detection and over-sampling methods. Studies also highlight the use of data augmentation, dropout, and cross-validation which stresses the idea to deal with imbalanced datasets and overfitting. Based on the review of different literature, there are still some drawbacks and weaknesses in the methods and techniques used for the analysis. As the potential benefits of computerized solutions for malignancy detection are tremendous, efficient algorithms can be identified and developed for routine usage in the diagnostic process by experts and clinicians.

\section{Future perspective}

This review provides an overview of the prominent number of publications related to image analysis and diagnosis of cervical Pap smear images. Cervical cell image segmentation has remained a significant challenge and is considered incomplete in addressing issues like debris removal, overlapped cytoplasm, and the poor of quality stain images. Another major challenge faced here is the availability of a sufficient number of real images for validation. The study can be focused on increasing the size of the dataset so that the accuracy of the computer-assisted diagnosing system can be made consistent with the images of different degrees of abnormality. As poor enhancement and segmentation result in poor results during the classification phase, efficient image enhancement and segmentation is an essential step in image analysis. Image analysis, storage, and throughput can be improved by making use of Graphical Processing Units (GPUs) that have a large number of dedicated parallel processing cores that make the system more efficient for image analysis. A new approach can be developed by combining advanced image processing techniques and $\mathrm{CNN}$ algorithms to create a better diagnostic system.

Hybrid-based techniques can be incorporated for better prediction of cancer diagnosis and treatment by combining other methods and techniques which will consequently increase the diagnostic sensitivity and specificity of cancer cell detection in pap smear screening. The new hybrid ensemble systems, which are a fusion of different "machine learning and deep learning" based algorithms, is another scope of work in cell image analysis. As the ensemble methods combine multiple learning algorithms, the wrongly classified instance can be corrected by another contributing algorithm. Thus, by incorporating novel methods, intelligent algorithms can be developed to improve the reliability of the support system in classifying a huge set of samples with good diagnostic sensitivity and specificity. The cervical cancer prediction model should be designed in such a way that it can be applied to diverse cancer datasets such as breast, liver, lung, thyroid, and kidney.

IoT (Internet of Things) can also be incorporated into the model so that patients at home can use personal health devices to notify health personnel in real time during emergency situations. Identifying better techniques for feature selection also strengthens the machine-learning process by reducing bias in the diagnosis. As accurate detection and classification seem to be a tedious task for a pathologist, especially during mass screening programs, the demand for a reliable automated detection system, expedites the need for automation in the diagnostic process. Future studies on cancer detection and diagnosis should concentrate more on the different model architectures that assist clinicians in choosing appropriate computer-assisted systems for diagnosis.

\section{Acknowledgments}

We would like to thank Dr. Ranjini Kudva, Professor, and all the staff in the Dept. of Pathology, Kasturba Medical College, Manipal Academy of Higher Education, Manipal, Karnataka, India for providing all support and guidance for the work. We also thank the Department of Pathology and Institutional Ethics Committee (IEC 472/2013) for providing permission to use the Images for the study.

\section{Conflict of interest}

There are no conflicts to declare.

\section{Supporting information}

Not Applicable. 


\section{References}

[1] L. Bruni, L. Barrionuevo-Rosas, G. Albero, M. Aldea, B. Serrano, S. Valencia, M. Brotons, M. Mena, R. Cosano, J. Mũnoz, F.X. Bosch, ICO/IARC Inf Cent HPV Cancer (HPV Inf Centre), 2019, 307, https://hpvcentre.net/statistics/reports/XWX.pdf.

[2] F. Bray, J. Ferlay, I. Soerjomataram, R. L. Siegel, L. A. Torre, A. Jemal, CA: A Cancer Journal for Clinicians, 2018, 68, 394 424, doi: 10.3322/caac.21492.

[3] H. Sung, J. Ferlay, R. L. Siegel, M. Laversanne, I. Soerjomataram, A. Jemal, F. Bray, CA: A Cancer Journal for Clinicians, 2021, 71, 209-249, doi: 10.3322/caac.21660.

[4] A. Volerman, A. S. Cifu, Journal of the American Medical Association (JAMA), 2014, 312, 2279-2280, doi: 10.1001/jama.2014.14992.

[5] R. A. Kerkar, Y. V. Kulkarni, The Journal of Obstetrics and Gynecology of India, 2006, 56, 115-122, Corpus ID: 74819514. [6] H. C. Kitchener, R. Blanks, H. Cubie, M. Desai, G. Dunn, R. Legood, A. Gray, Z. Sadique, S. Moss, Clinical Governance: an International Journal, 2011, 16, doi: 10.1108/cgij.2011.24816caa.003.

[7] R. Sankaranarayanan, Annals of Global Health, 2014, 80, 412, doi: 10.1016/j.aogh.2014.09.014.

[8] C. O'Connor, J. U. Adams, Essentials of Cell Biology, Cambridge, MA: NPG Education, 2010.

[9] L. L. Rubin, K. L. Philpott, S. F. Brooks, Current Biology, 1993, 3, 391-394, doi: 10.1016/0960-9822(93)90211-6.

[10] R. L. Ehrmann, Comprehensive Therapy, 1996, 22, 67-75, PMID: 8689865.

[11] J. Ferlay, I. Soerjomataram, R. Dikshit, S. Eser, C. Mathers, M. Rebelo, D. M. Parkin, D. Forman, F. Bray, International Journal of Cancer, 2015, 136, E359-E386, doi: 10.1002/ijc.29210.

[12] Z E. J. Mariarputham, A. Stephen, Computational and Mathematical Methods in Medicine, 2015, 586928, doi: $10.1155 / 2015 / 586928$.

[13] J. Jantzen, G. Dounias, Proceedings of the Nature-Inspired Smart Information Systems 2nd Annual Symposium, 2006, 10, $1-$ 11.

[14] T. Conceição, C. Braga, L. Rosado, M. J. M. Vasconcelos, International Journal of Molecular Sciences, 2019, 20, 5114, doi: 10.3390/ijms20205114.

[15] Z. Lu, G. Carneiro, A. P. Bradley, IEEE Transactions on Image Processing, 2015, 24, 1261-1272, doi: 10.1109/tip.2015.2389619.

[16] X. Li, Z. Xu, X. Shen, Y. Zhou, B. Xiao, T. Q. Li, Current Oncology, 2021, 28, 3585-3601, doi: 10.3390/curroncol28050307.

[17] P. B. Shanthi, F. Faruqi, K. S. Hareesha, R. Kudva, Asian Pacific Journal of Cancer Prevention, 2019, 20, 3447, doi:
10.31557/APJCP.2019.20.11.3447

[18] E. F. Arriaga-Garcia, R. E. Sanchez-Yanez, J. Ruiz-Pinales, M. de Guadalupe Garcia-Hernandez, Journal of Electronic Imaging, 2015, 24, 053009, doi: 10.1117/1.jei.24.5.053009.

[19] H. Ibrahim, N. S. P. Kong, IEEE Transactions on Consumer Electronics, 2007, 53, 1752-1758, doi: 10.1109/TCE.2007.4429280.

[20] D. Vendhan, R. Selvakumar, IEEE International Conference on Computational Intelligence and Computing Research, 2010.

[21] S. Kaur, P. Kaur, International Journal of Computer Applications Technology and Research, 2015, 4, 414-418, doi: 10.7753/IJCATR0405.1016.

[22] T. Guan, D. Zhou, W. Fan, K. Peng, C. Xu, X. Cai, IEEE International Conference on Robotics and Biomimetics, 2014, 107-112, doi: 10.1109/ROBIO.2014.7090315.

[23] Y. S. Choi, R. Krishnapuram, IEEE Transactions on Image Processing, 1997, 6, 808-825, doi: 10.1109/83.585232.

[24] S. Hashemi, S. Kiani, N. Noroozi, M. E. Moghaddam, Pattern Recognition Letters, 2010, 31, 1816-1824, doi: 10.1016/j.patrec.2009.12.006.

[25] A. Ghaheri, S. Shoar, M. Naderan, S. S. Hoseini, Oman Medical Journal, 2015, 30, 406-416, doi: 10.5001/omj.2015.82.

[26] L. Tong, J. Wang, S. Li, K. Xiao, Q. Peng, Computer Modelling and New Technologies, 2015, 19, 7-13.

[27] S. Gopinathan, M. S. Gayathri, International Journal of Advanced Engineering Research and Science, 2016, 3, 236818 , doi: $10.1145 / 3301506.3301539$.

[28] V. Magudeeswaran, C. Ravichandran, Mathematical Problems in Engineering, 2013, 891864, doi: $10.1155 / 2013 / 891864$.

[29] T. Deepa A. N. Rao, Indian Journal of Science and Technology, 2016, 9, 1-10, doi: 10.17485/ijst/2016/v9i45/96623.

[30] J. R. Tang, N. A. Mat Isa, Computers \& Electrical Engineering, 2014, 40, 86-103, doi: 10.1016/j.compeleceng.2014.05.017.

[31] H. Vidyasaraswathi, M. Hanumantharaju, International Conference on Advanced Research in Computer Science Engineering \& Technology, 2015, 48, 1-6, doi: 10.1145/2743065.2743113.

[32] U. Kumbhar, V. Patil, S. Rudrakshi, International Journal of Engineering Research and Technology, 2013, 2, 2359-2364.

[33] D. Sheet, H. Garud, A. Suveer, M. Mahadevappa, J. Chatterjee, IEEE Transactions on Consumer Electronics, 2010, 56, 2475-2480, doi: 10.1109/tce.2010.5681130.

[34] G. Li, Y. Tong, X. Xiao, Procedia Engineering, 2011, 15, 1590-1594, doi: 10.1016/j.proeng.2011.08.296.

[35] J. R. Tang, N. A. Mat Isa, E. S. Ch’ng, PLoS One, 2015, 10, e0142830, doi: 10.1371/journal.pone.0142830.

[36] D. Surya Prabha, J. Satheesh Kumar, Wireless Personal 
Communications, 2017, 93, 223-244, doi: 10.1007/s11277-0163536-x.

[37] T. Chankong, N. Theera-Umpon, S. Auephanwiriyakul, Computer Methods and Programs in Biomedicine, 2014, 113, 539-556, doi: 10.1016/j.cmpb.2013.12.012.

[38] J. R. Tang, N. A. M. Isa, E. S. Ch'ng, IEEE International Conference on Control System, Computing and Engineering (ICCSCE), 2015, 111-116, doi: 10.1109/ICCSCE.2015.7482168. [39] R. Saha, M. Bajger, G. Lee, International conference on digital image computing: techniques and applications (DICTA). IEEE, 2016, 1-8, doi: 10.1109/DICTA.2016.7797086.

[40] S. Wienert, D. Heim, K. Saeger, A. Stenzinger, M. Beil, P. Hufnagl, M. Dietel, C. Denkert, F. Klauschen, Scientific Reports, 2012, 2, 503, doi: 10.1038/srep00503.

[41] J. Talukdar, C. K. Nath, P. H. Talukdar, markers, 2013, 3, $460-462$.

[42] T. F. Chan, L. A. Vese, Conference Record of the ThirtyFourth Asilomar Conference on Signals, Systems and Computers (Cat. No. 00CH37154), IEEE, 2000, 1, 490-494, doi: 10.1109/ACSSC.2000.911004.

[43] T. F. Chan, L. A. Vese, IEEE Transactions on Image Processing, 2001, 10, 266-277, doi: 10.1109/83.902291.

[44] T. Liu, H. Xu, W. Jin, Z. Liu, Y. Zhao, W. Tian, Computational and Mathematical Methods in Medicine, 2014, 110, doi: 10.1155/2014/890725.

[45] A. Pratondo, C. K. Chui, S. H. Ong, Journal of Visual Communication and Image Representation, 2017, 43, 1-9, doi: 10.1016/j.jvcir.2016.11.019.

[46] P. T. T. Aung, A. S. Khaing, H. M. Tun, International Journal of Scientific \& Technology Research, 2016, 5, 92-97, Corpus ID: 4657604.

[47] B. Saini, G. Sethi, International Journal of Research in Engineering and Technology, 2013, 2, 2319-1163, doi: 10.15623/IJRET.2013.0204028.

[48] F. M. Mohsen, M. M. Hadhoud, K. Amin, International Journal of Advanced Computer Science and Applications (IJACSA), 2011, 1-9, doi: 10.14569/SpecialIssue.2011.010102.

[49] P. Sukumar, R. K. Gnanamurthy, ABI Genetika, 2015, 47, 863-876, doi: 10.2298/gensr1503863s.

[50] S. Cheng, S. Liu, J. Yu, G. Rao, Y. Xiao, W. Han, W. Zhu, X. Lv, N. Li, J. Cai, Z Wang, X. Feng, F. Yang, X. Geng, J. Ma, X. Li, Z. Wei, X. Zhang, T. Quan, S. Zeng, L. Chen, J. Hu, X. Liu, Nature Communications, 2021, 12, 5639., doi: 10.1038/s41467021-25296-x.

[51] S. Gautam, A. Bhavsar, A. K. Sao. K. K. Harinarayan, Medical Imaging 2018: Digital Pathology. International Society for Optics and Photonics, 2018, 10581:105810X, doi: $10.1117 / 12.2293526$

[52] M. Zhao, H. Wang, Y. Han, X. Wang, H.-N. Dai, X. Sun, J.
Zhang, M. Pedersen, Future Generation Computer Systems, 2021 114, 185-194, doi: 10.1016/j.future.2020.07.045

[53] J. Huang, T. Wang, D. Zheng, Y. He, Bioengineered, 2020, 11, 484-501, doi: 10.1080/21655979.2020.1747834.

[54] T. Wang, J. Huang, D. Zheng, Y. He, IEEE Access, 2020, 8, 75846-75859, doi: 10.1109/access.2020.2989369.

[55] M. Wu, C. Yan, H. Liu, Q. Liu, Y. Yin, Bioscience Reports, 2018, 38, BSR20181769, doi: 10.1042/bsr20181769.

[56] W. Mousser, S. Ouadfel, ICCTA '19: Proceedings of the 2019 5th International Conference on Computer and Technology Applications, 2019, 6-10, doi: 10.1145/3323933.3324060.

[57] Y. Jusman, S. C. Ng, N. A. Abu Osman, The Scientific World Journal, 2014, 810368, doi: 10.1155/2014/810368.

[58] K. Bora, M. Chowdhury, L. B. Mahanta, M. K. Kundu, A. K. Das, Computer Methods and Programs in Biomedicine, 2017, 138, 31-47, doi: 10.1016/j.cmpb.2016.10.001.

[59] C. N. Rao, S. S. Sastry, K. Mallika, H. S. Tiong, K. Mahalakshmi, International Journal of Innovative Research in Science, Engineering and Technology, 2013, 2, 4531-4538.

[60] S. S. Sastry, K. Mallika, B. G. S. Rao, S. T. Ha, S. Lakshminarayana, Phase transitions, 2012, 85, 735-749, doi: 10.1080/01411594.2012.664275.

[61] R. M. Haralick, K. Shanmugam, I. Dinstein, IEEE Transactions on Systems, Man, and Cybernetics, 1973, SMC-3, 610-621, doi: 10.1109/tsmc.1973.4309314.

[62] L. B. Mahanta, K. Bora, International Journal of Image Processing, Signal processing and Pattern Recognition, 2013, 6, 451-456, Corpus ID: 14703189.

[63] K. Krishnaveni, S. Allwin, S. P. K. Kenny, G. Mariappan, International Conference on Computational Intelligence and Computing Research. IEEE, 2010, 1-4, doi: 10.1109/ICCIC.2010.5705914.

[64] G. Castellano, L. Bonilha, L. M. Li, F. Cendes, Clinical Radiology, 2004, 59, 1061-1069, doi: 10.1016/j.crad.2004.07.008.

[65] A. Baraldi F. Parmiggiani, IEEE Transactions On Geoscience And Remote Sensing, 1995, 33, 293-304, doi:10.1109/36.377929.

[66] L. Nanni, S. Brahnam, S. Ghidoni, E. Menegatti, T. Barrier, PLoS One, 2013, 8, e83554, doi: 10.1371/journal.pone.0083554.

[67] K. Soumya, K. Sneha, C. Arunvinodh, Biomedical and Pharmacology Journal, 2016, 9, 663-671, doi: 10.13005/bpj/988. [68] M. A. Devi, S. Ravi, J. Vaishnavi, S. Punitha, Procedia Computer Science, 2016, 89, 465-472, doi: 10.1016/j.procs.2016.06.105.

[69] M.-H. Tsai, Y.-K. Chan, Z.-Z. Lin, S.-F. Yang-Mao, P.-C. Huang, Pattern Recognition Letters, 2008, 29, 1441-1453, doi: 10.1016/j.patrec.2008.02.024

[70] J. N. Kapur, P. K. Sahoo, A. K. C. Wong, Computer Vision, Graphics, and Image Processing, 1985, 29, 273-285, doi: 10.1016/0734-189x(85)90125-2. 
[71] S Cheng, S Liu, J Yu J, G Rao, Y Xiao, W Han, W Zhu, X Lv, N Li, J Cai, Z Wang, Natare Communivcation, 2021, 12, 110, doi: 10.1038/s41467-021-25296-X.

[72] V. Chandran, M. G. Sumithra, A. Karthick, T. George, M. Deivakani, B. Elakkiya, U. Subramaniam, S. Manoharan, BioMed Research International, 2021, 2021, 1-15, doi: $10.1155 / 2021 / 5584004$.

[73] M. M. Rahaman, C. Li, Y. Yao, F. Kulwa, X. Wu, X. Li, Q. Wang, Computers in Biology and Medicine, 2021, 36, 104649, doi: 10.1016/j.compbiomed.2021.104649.

[74] J. Zhang, Y. Liu, Medical Image Computing and ComputerAssisted Intervention - MICCAI, 2004, 873-880, doi: 10.1007/978-3-540-30136-3_106.

[75] Y.-F. Chen, P.-C. Huang, K.-C. Lin, H.-H. Lin, L.-E. Wang, C.-C. Cheng, T.-P. Chen, Y.-K. Chan, J. Y. Chiang, IEEE Journal of Biomedical and Health Informatics, 2014, 18, 94-108, doi: 10.1109/jbhi.2013.2250984.

[76] S. Athinarayanan, M. Srinath, R. Kavitha, ICTACT Journal on Image and Video Processing, 2016, 6. 1244-1251, doi: 10.21917/ijivp.2016.0181.

[77] Q. Miao, J. Derbas, A. Eid, H. Subramanian, V. Backman, BioMed Research International, 2016, 2016:6090912, doi: 10.1155/2016/6090912.

[78] M. E. Plissiti, C. Nikou, A. Charchanti, Pattern Recognition Letters, 2011, 32, 838-853, doi: 10.1016/j.patrec.2011.01.008.

[79] L. Wei, Q. Gan, T. Ji, Computer-assisted surgery, 2017, 22, 186-199, doi: 10.1080/24699322.2017.1389397.

[80] B. Taha, J. Dias, N. Werghi, Annual Conference on Medical Image Understanding and Analysis, Springer, 2017, 261-272, doi: 10.1007/978-3-319-60964-5 23.

[81] I. U. Khan, N. Aslam, R. Alshehri, S. Alzahrani, M. Alghamdi, A. Almalki, M. Balabeed, Scientific Programming, 2021, 2021, 1-10, doi: 10.1155/2021/5540024.

[82] W. Chen, X. Li, L. Gao, W. Shen, Applied Sciences, 2020, 10, 7292, doi: 10.3390/app10207292.

[83] D. N. Diniz, M. T. Rezende, A. G. C. Bianchi, C. M. Carneiro, E. J. S. Luz, G. J. P. Moreira, D. M. Ushizima, F. N. S. de Medeiros, M. J. F. Souza, Journal of Imaging, 2021, 7, 111, doi: 10.3390/jimaging 7070111.

[84] M. F. Ijaz, M. Attique, Y. Son, Sensors, 2020, 20, 2809, doi: $10.3390 / \mathrm{s} 20102809$.

[85] S. Jahan, M. D. S. Islam, L. Islam, T. Y. Rashme, A. A. Prova, B. K. Paul, M. D. M. Islam, M. K. Mosharof, SN Applied Sciences, 2021, 3, 806, doi: 10.1007/s42452-021-04786-z.

[86] M. M. Ali, K. Ahmed, F. M. Bui, B. K. Paul, S. M. Ibrahim, J. M. W. Quinn, M. A. Moni, Computers in Biology and Medicine, 2021, 139, 104985, doi: 10.1016/j.compbiomed.2021.104985.

[87] K. S. Alqahtani, S. Srinivasan, D. P. Mital, International Journal of Medical Engineering and Informatics, 2017, 9, 61-72, doi: 10.1504/IJMEI.2017.080925.

[88] Y. LeCun, Y. Bengio, G. Hinton, Nature, 2015, 521, 436-444, doi: $10.1038 /$ nature 14539 .

[89] L. Zhang, L. Lu, I. Nogues, R. M. Summers, S. Liu, J. Yao, IEEE Journal of Biomedical and Health Informatics, 2017, 21,
1633-1643, doi: 10.1109/jbhi.2017.2705583.

[90] D. Ravi, C. Wong, F. Deligianni, M. Berthelot, J. AndreuPerez, B. Lo, G.-Z. Yang, IEEE Journal of Biomedical and Health Informatics, 2017, 21, 4-21, doi: 10.1109/jbhi.2016.2636665.

[91] H.-C. Shin, H. R. Roth, M. Gao, L. Lu, Z. Xu, I. Nogues, J. Yao, D. Mollura, R. M. Summers, IEEE Transactions on Medical Imaging, 2016, 35, 1285-1298, doi: 10.1109/tmi.2016.2528162.

[92] M. Anthimopoulos, S. Christodoulidis, L. Ebner, A. Christe, S. Mougiakakou, IEEE Transactions on Medical Imaging, 2016, 35, 1207-1216, doi: 10.1109/tmi.2016.2535865.

[93] J. Hyeon, H.-J. Choi, K. N. Lee, B. D. Lee, 18th IEEE International Conference on Mobile Data Management (MDM), 2017, 382-385, doi: 10.1109/MDM.2017.66.

[94] D. Mundhra, B. Cheluvaraju, J. Rampure, T. Rai Dastidar, Deep Learning in Medical Image Analysis and Multimodal Learning for Clinical Decision Support, 2017, 178-185, doi: 10.1007/978-3-319-67558-9 21.

[95] AU Rehman, N Ali, I Taj, M Sajid, KS Karimov, Mathematical Problems in Engineering, 2020, 4864835, doi: 10.1155/2020/4864835.

[96] M. A. Mohammed, F. Abdurahman, Y. A. Ayalew, BMC Biomedical Engineering, 2021, 3, 11, doi: 10.1186/s42490-02100056-6.

[97] Z. Lu, G. Carneiro, A. P. Bradley, D. Ushizima, M. S. Nosrati, A. G. C. Bianchi, C. M. Carneiro, G. Hamarneh, IEEE Journal of Biomedical and Health Informatics, 2017, 21, 441-450, doi: 10.1109/jbhi.2016.2519686.

[98] K. Pedersen, E. A. Burger, S. Campbell, M. Nygård, E. Aas, S. Lönnberg, European Journal of Public Health, 2017, 27, 10891094, doi: 10.1093/eurpub/ckx073.

[99] N. Razali, S. A. Mostafa, A. Mustapha, M. H. A. Wahab, N. A. Ibrahim, Journal of Physics: Conference Series, 2020, 1529, 022102, doi: 10.1088/1742-6596/1529/2/022102

[100] L. Mredhula, M. Dorairangaswamy, International Journal of Medical Engineering and Informatics, 2017, 9, 30-46, doi: 10.1504/IJMEI.2017.10000840.

[101] S. Athinarayanan, D. M. V. Srinath, R. Kavitha, IOSR Journal of Computer Engineering, 2017, 19, 23-34, doi: 10.9790/0661-1901042334.

[102] A. Qayyum, S. M. Anwar, M. Awais, M. Majid, Neurocomputing, 2017, 266, 8-20, doi: 10.1016/j.neucom.2017.05.025.

[103] K. Suzuki, Radiological Physics and Technology, 2017, 10, 257-273, doi: 10.1007/s12194-017-0406-5.

[104] S. Sornapudi, R. J. Stanley, W. V. Stoecker, H. Almubarak, R. Long, S. Antani, G. Thoma, R. Zuna, S. R. Frazier, Journal of Pathology Informatics, 2018, 9, 5, doi: 10.4103/jpi.jpi 7417 [105] H Wieslander, G Forslid, E Bengtsson, C Wahlby, JM Hirsch, C Runow Stark, S Kecheril Sadanandan, Proceedings of the IEEE International Conference on Computer Vision Workshops, 2017, 17522158, doi: 10.1109/ICCVW.2017.18.

[106] A. Sarwar, J. Suri, M. Ali, V. Sharma, Journal of Ambient Intelligence and Humanized Computing, 2016, 7, 593-606, doi: 10.1007/s12652-016-0353-8.

[107] H.-L. Shen, J. H. Xin, Journal of Electronic Imaging, 2005, 
14, 33003, doi: 10.1117/1.1990007.

[108] B. Sharma, K. K. Mangat, International Journal of Computers and Applications, 2016, 147, 16-20, doi:10.5120/ijca2016911170.

[109] D. A. Clausi, B. Yue, IEEE Transactions on Geoscience and Remote Sensing, 2004, 2, 215-228, doi: 10.1109/TGRS.2003.817218.

[110] W. William, A. Ware, A. H. Basaza-Ejiri, J. Obungoloch, Computer Methods and Programs in Biomedicine, 2018, 164, 1522, doi: 10.1016/j.cmpb.2018.05.034.

[111] M. E. Plissiti, C. Nikou, A. Charchanti, IEEE Transactions on Information Technology in Biomedicine, 2011, 15, 233-241, doi: 10.1109/titb.2010.2087030.

[112] RU Deepak, RR Kumar, NB Byju, PN Sharathkumar, C Pournami, S Sibi, E Bengtsson, K Sujathan, Journal of Cytology and Histology, 2015, 3, 20-23, doi: 10.4172/2157-7099.S3-010.

\section{Author Information}

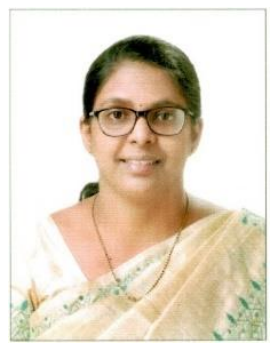

Dr. Shanthi $\boldsymbol{P} \boldsymbol{B}$ is an Assistant Professor in the Department of Computer Science and Engineering, Manipal Institute of Technology, Karnataka, India. She obtained her PhD in Medical Image Processing from Manipal Academy of Higher Education, Karnataka, India in 2020. Her research interest includes computer vision, medical image processing, health care diagnosis, automation, machine learning and artificial intelligence. She is a life member of Indian Society of Systems for Science and Engineering (ISSE).

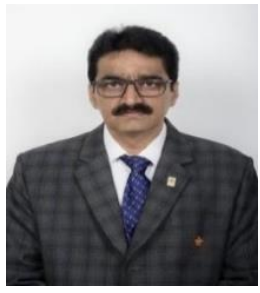

Dr. Hareesha $\boldsymbol{K} S$ is a Professor in the Department of Computer Applications at Manipal Institute of Technology, Manipal Academy of Higher Education, Karnataka, India. He received his $P h D$ in Computer Science and Engineering, Kuvempu University in Karnataka,India in 2008. His research interest focuses on improving the machine learning algorithms, design of intelligent soft computing models in digital image. He is a member in professional bodies IEEE (senior member), MISTE, IACSIT and hold research funded projects from DST, New Delhi and start-up funded by DST-BIRAC, Government of India.

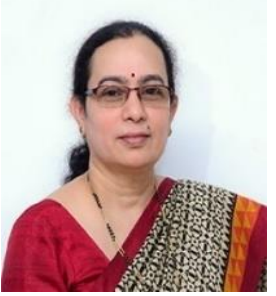

Dr. Ranjini Kudva is a Professor in the Department of Pathology, Kasturba Medical College, Manipal. She obtained her MBBS from Calicut Medical College, Calicut University in 1987 and MD Pathology from Kasturba Medical College, Mangalore University in 1993. Her research interests are Oncopathology, Gynaec pathology, Paediatric pathology, and Hemato oncology. She is a life member of Indian association of Pathologists and Microbiologist (IAPM) and Indian Academy of Cytologists (IAC).

Publisher's Note: Engineered Science Publisher remains neutral with regard to jurisdictional claims in published maps and institutional affiliations. 